\title{
A micro-analytical approach to partition coefficients in plagioclase and clinopyroxene of basaltic sills in Serra Geral Formation, Paraná Basin, Brazil
}

\author{
Leonardo Cardoso Renner', Léo Afraneo Hartmann², Wilson Wildner³, Hans-Joachim \\ Massonne $^{4}$ \& Thomas Theye ${ }^{4}$
}

\begin{abstract}
In the present study, detailed micro-analytical data (major and trace elements) in minerals from sills of Serra Geral Formation represents the first attempt to evaluate the partition coefficient of Sc, Ti, V, Cr, Co, $\mathrm{Ni}, \mathrm{Cu}, \mathrm{Zn}, \mathrm{Rb}, \mathrm{Sr}, \mathrm{Y}, \mathrm{Zr}, \mathrm{Nb}, \mathrm{Mo}, \mathrm{Cs}, \mathrm{Ba}, \mathrm{REE}, \mathrm{Hf}, \mathrm{Ta}, \mathrm{Pb}$, Th and $\mathrm{U}$ among coexisting plagioclase, augite and pigeonite from 12 tholeiitic basaltic sills along the eastern border of the Paraná Basin, Brazil. These 12 samples come from Rio Grande do Sul, Paraná, São Paulo and Goiás states. Plagioclase/melt partition coefficients $\mathrm{K}_{\mathrm{D}}$ were determined in core, intermediate and rim zones using LA-ICP-MS and the results were compared with the variations of the elements $\mathrm{Ca}, \mathrm{Al}, \mathrm{Na}$ and $\mathrm{Si}$ in those areas. Partition coefficients of $\mathrm{Sr}$ and $\mathrm{Eu}$ decrease with the increase of $\mathrm{Ca}$ concentration in plagioclase. A reduction in temperature and consequently a rise in $\mathrm{Na}$ and $\mathrm{Si}$ levels increase the partition coefficients of $\mathrm{Sr}$ and $\mathrm{Eu}$ at the rim of mineral The $\mathrm{K}_{\mathrm{D}}$ of $\mathrm{Ni}$ and $\mathrm{V}$ in clinopyroxene has positive correlations with $\mathrm{Mg}^{2+}, \mathrm{Ca}^{2+}$ and $\mathrm{Al}^{3+}$ due to higher concentrations of those elements at the core and lower concentration towards the rim and negative correlations of $\mathrm{K}_{\mathrm{D}}$ of $\mathrm{Sc}, \mathrm{Cr}, \mathrm{Co}, \mathrm{Pb}$ and $\mathrm{Lu}$, due to preferential substitutions by $\mathrm{Fe}^{2+}$ in sixfold coordination. The use of LA-ICP-MS and EPMA made possible the chemical quantification of major and trace elements and the determination of partition coefficients in coexisting plagioclase and clinopyroxene in basaltic melts.
\end{abstract}

Keywords: Partition coefficient, plagioclase, clinopyroxene, Serra Geral Formation, LA-ICP-MS.

Resumo Uma abordagem microanalítica dos coeficientes de partição em plagioclásio e clinopiroxênio de soleiras basálticas da Formação Serra Geral, Bacia do Paraná, Brasil. No presente estudo, dados detalhados de microanálise (elementos maiores e traços) em minerais de sills da Formação Serra Geral representam a primeira tentativa de avaliar o coeficiente de partição de $\mathrm{Sc}, \mathrm{Ti}, \mathrm{V}, \mathrm{Cr}, \mathrm{Co}, \mathrm{Ni}, \mathrm{Cu}, \mathrm{Zn}, \mathrm{Rb}, \mathrm{Sr}, \mathrm{Y}, \mathrm{Zr}, \mathrm{Nb}, \mathrm{Mo}, \mathrm{Cs}$, $\mathrm{Ba}$, REE, $\mathrm{Hf}, \mathrm{Ta}, \mathrm{Pb}$, Th e U entre plagioclásio, augita e pigeonita coexistentes em 12 sills basálticos toleíticos ao longo da borda leste da Bacia do Paraná, Brasil. Estas 12 amostras são dos estados do Rio Grande do Sul, Paraná, São Paulo e Goiás. Coeficientes de partição em plagioclásio foram comparados em zonas de núcleo, intermédio e borda, usando LA-ICP-MS, e os resultados foram comparados com as variações das áreas de distribuição dos elementos $\mathrm{Ca}, \mathrm{Al}, \mathrm{Na}$ e Si. O coeficiente de partição do $\mathrm{Sr}$ e Eu diminui com o aumento da concentração de Ca no plagioclásio e aumenta para a borda, relacionado com o aumento da concentração de $\mathrm{Na}$ e Si e diminuição da temperatura. $\mathrm{O} \mathrm{K}_{\mathrm{D}}$ do Ni e $\mathrm{V}$ nos clinopiroxênios analisados possui correlações positivas para $\mathrm{Mg}^{2+}, \mathrm{Ca}^{2+} \mathrm{e} \mathrm{Al}^{3+}$ devido a altas concentrações destes elementos no núcleo, diminuindo para a borda, e correlações negativas de $\mathrm{K}_{\mathrm{D}} \mathrm{Sc}, \mathrm{Cr}, \mathrm{Co}, \mathrm{Pb}$ e Lu, devido a substituição preferencial pelo $\mathrm{Fe}^{2+}$ em coordenações octaédricas. O uso de LA-ICP-MS e EPMA possibilitam a caracterização química de elementos maiores e traços e a determinação do coeficiente de partição em minerais coexistentes e o líquido.

Palavras-chave: Coeficiente de partição, plagioclásio, clinopiroxênio, Formação Serra Geral, LA-ICP-MS.

INTRODUCTION Continental flood basalts are major igneous units and their investigation enables to understand the processes active in the mantle and crust. The Serra Geral Formation of South America comprises the Paraná magmatic province, constituted by basalts, basaltic andesites and acid rocks. Recent technological advances, such as the spot LA-ICP-MS analysis of selected small portions of crystals, have opened a window into processes related to the crystallization of the magmas. This is a detailed investigation of trace element content and zonation in plagioclase and clinopyroxene from Paraná province sills and dykes and of their partition coefficients with the magmatic liquid.

Micro-analytical data (EPMA and LA-ICP-MS) and partitioning coefficients of $\mathrm{Sc}, \mathrm{Ti}, \mathrm{V}, \mathrm{Cr}, \mathrm{Co}, \mathrm{Ni}$, $\mathrm{Cu}, \mathrm{Zn}, \mathrm{Rb}, \mathrm{Sr}, \mathrm{Y}, \mathrm{Zr}, \mathrm{Nb}$, Mo, Cs, Ba, REE, Hf, Ta, $\mathrm{Pb}$, Th and $\mathrm{U}$ between plagioclase and clinopyroxene in basalt and andesite basaltic melts is here intensively

1 - Programa de Pós-graduação em Geociências, Instituto de Geociências, Universidade Federal do Rio Grande do Sul, Porto Alegre (RS), Brazil. E-mail: leorenner@gmail.com

2 - Instituto de Geociências, Universidade Federal do Rio Grande do Sul, Porto Alegre (RS), Brazil. E-mail: leo.hartmann@ufrgs.br

3 - CPRM/SUREG-PA, Serviço Geológico do Brasil, Porto Alegre (RS), Brazil. E-mail: wwildner@pa.cprm.gov.br

4 - Institut für Mineralogie und Kristallchemie, Stuttgart Universität, Germany. E-mail: h-j.massone@mineralogie.uni-stuttgart.de,

thomas.theye@mineralogie.uni-stuttgart.de 
used in sills of Serra Geral Formation, Paraná Basin, Brazil (Fig. 1). We also utilize chemical data to examine how variations in major components in the plagioclase and clinopyroxene influence $\mathrm{K}_{\mathrm{D}}$ in basalt and basaltic andesite melts.

Plagioclase is affected by rebalancing of $\mathrm{Ca}^{2+} \mathrm{Al}^{3+}$ $\leftrightarrow \mathrm{Na}^{+} \mathrm{Si}^{4+}$ influencing in partition coefficients such as $\mathrm{Sr}, \mathrm{Eu}$ and $\mathrm{Pb}$ in core, intermediate and rim zones. Clinopyroxene exerts major control on distributions of some trace elements and REE in substitutions of $\mathrm{Fe}$ and $\mathrm{Mg}$ (sixfold coordination), $\mathrm{Ca}$ (eightfold coordination) and $\mathrm{Al}$ (six or fourfold coordination).
The recent advances of micro-analytical techniques allow to determine the concentration of major, trace and REE elements in diversity zones of minerals, characterizing the chemical order of crystallization and observing probable variables that can influence in mineral/melt partition coefficients such as $T, P, f \mathrm{O}_{2}$ and bulk composition.

ANALYTICAL METHODS Sampleswerecollected during field trips in Rio Grande do Sul state, Brazil by CPRM (Geological Survey of Brazil) projects in the last five years. These samples are representative of sills in

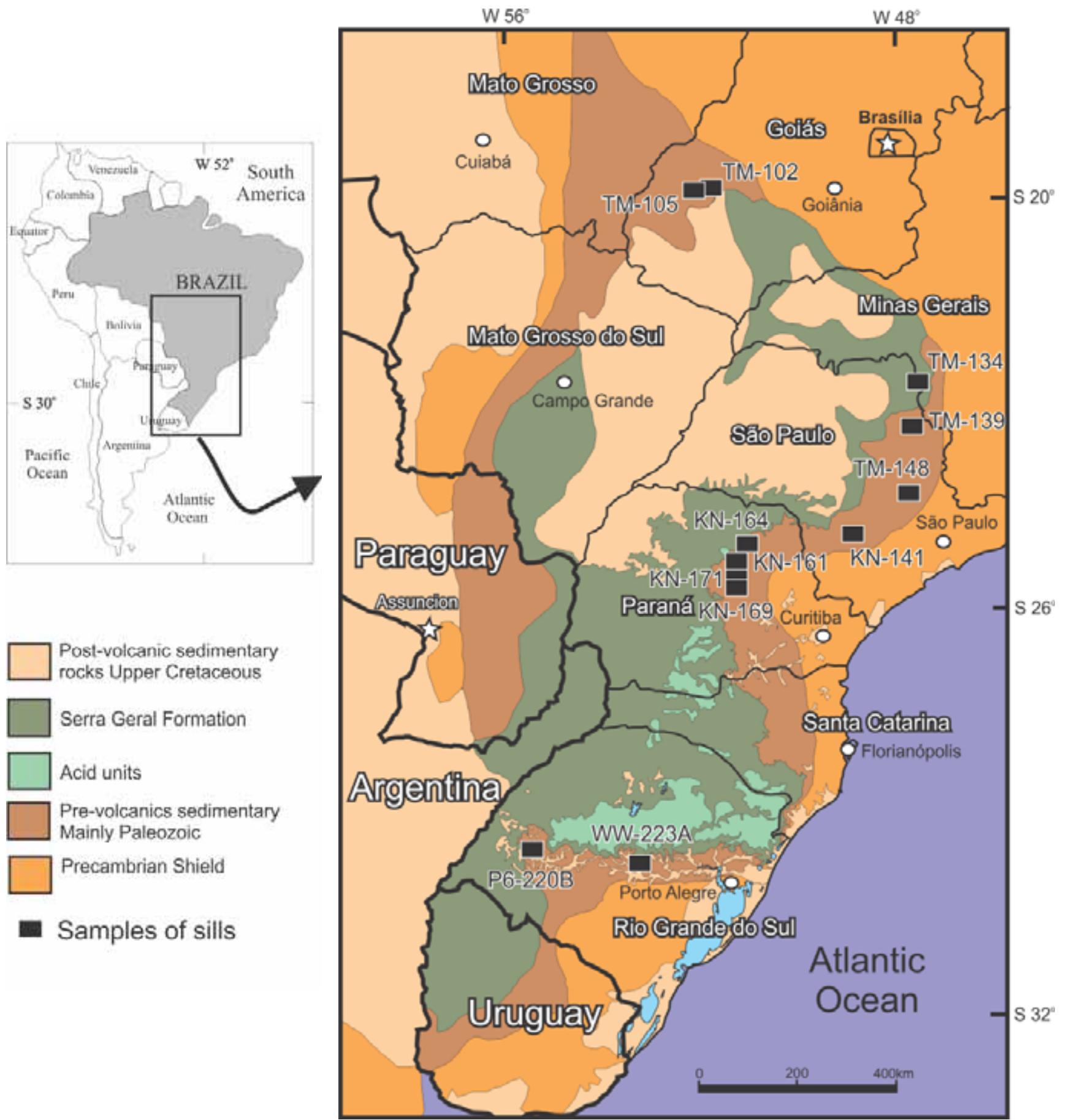

Figure 1 - Geological map showing the general rocks and distribution of sill samples of Paraná Basin in South America. Modified from Bellieni et al. (1986), Melfi et al. (1988), Nardy et al. (2002) and Machado (2003). 
the Santiago and Alegrete regions, west of Rio Grande do Sul and sills of Agudo region, central Rio Grande do Sul. The selected samples consist of two sills from Rio Grande do Sul. The other ten sills samples collected by CPRM are from Paraná, São Paulo and Goiás states in the Paraná Basin (Fig. 1).

Major and trace elements were analyzed in whole rocks by Inductively Coupled Plasma - Emission Spectrometer (ICP-ES) and Inductively Coupled Plasma - Mass Spectrometer (ICP-MS) at Acme Analytical Laboratories Ltd. in Canada. Chemical data of the analyzed samples are shown in table 1 .

Micro-analyses of plagioclase and clinopyroxene of 12 samples of sills of the Paraná Basin were performed at the Institut für Mineralogie und Kristallchemie of the Universität Stuttgart in Germany. These analyses were conducted in thin sections coated by a thin film of carbon and inserted two by two and analyzed by a CAMEXA SX-100 probe, which is equipped with five highresolution wavelength-dispersive spectrometer (WDS) and one low-resolution energy-dispersive spectrometer (EDS). Analytical conditions for plagioclase and clinopyroxene included a focused beam (beam diameter $\sim 1 \mu \mathrm{m})$, Faraday current of $15 \mathrm{nA}$ with an acceleration voltage of $15 \mathrm{kV}$. Spectrometer SP1 - TAP (Al and $\mathrm{Si})$, SP2 - LLIF (Fe, Ni and Mn), SP3 - LPET (Ti and Cr), SP4 - TAP (Na and Mg) and SP5 - PET (K and $\mathrm{Ca}$ ) and calibration standards were: Diopside for $\mathrm{Si}, \mathrm{Mg}$ and $\mathrm{Ca}$; Cordierite for $\mathrm{Al} ; \mathrm{Fe}_{2} \mathrm{O}_{3}$ for $\mathrm{Fe}$; $\mathrm{NiO}$ for $\mathrm{Ni}$; Rutile for Ti; $\mathrm{Cr}_{2} \mathrm{O}_{3}$ for $\mathrm{Cr}$; Rhodochrosite for Mn; Albite for $\mathrm{Na}$ and Orthoclase for K. Counting times were $20 \mathrm{~s}$ on the peak for all these elements except $\mathrm{Na}$ with $10 \mathrm{~s}$. Background counting time was $20 \mathrm{~s}$ for $\mathrm{Al}, \mathrm{Si}, \mathrm{Mn}$ and $\mathrm{Fe}, 10 \mathrm{~s}$ for $\mathrm{Ni}, \mathrm{Ti}, \mathrm{Cr}, \mathrm{Mg}, \mathrm{K}$ and $\mathrm{Ca}$ and $10 \mathrm{~s}$ for $\mathrm{Na}$ on each side of the background.

For the 12 samples, over 184 backscattering electron images were acquired by CAMECA SX-100 in the same laboratory. The images were acquired at $15 \mathrm{kV}$ and $15 \mathrm{nA}$, modifying the brightness, gamma and contrast to accentuate the textures in individual crystals. The elements analyzed in plagioclase and clinopyroxene crystals, including portions of the matrix, were: $\mathrm{K} 2 \mathrm{O}, \mathrm{FeO}, \mathrm{Na}_{2} \mathrm{O}, \mathrm{Al}_{2} \mathrm{O}_{3}, \mathrm{TiO}_{2}, \mathrm{MnO}$, $\mathrm{CaO}, \mathrm{Cr}_{2} \mathrm{O}_{3}, \mathrm{NiO}, \mathrm{MgO}$ and $\mathrm{SiO}_{2}$, totalizing about 680 analyzed points, time analyses $2.5 \mathrm{~min}$ each.

Also characteristic X-ray distribution maps were made of $\mathrm{K}, \mathrm{Na}, \mathrm{Ca}, \mathrm{Fe}, \mathrm{Mg}$, Ti and $\mathrm{Al}$ for plagioclase and clinopyroxene in each sample, totaling 18 maps.

A total of 217 spots were analyzed for Sc, Ti, V, Cr, $\mathrm{Mn}, \mathrm{Co}, \mathrm{Ni}, \mathrm{Cu}, \mathrm{Zn}, \mathrm{Rb}, \mathrm{Sr}, \mathrm{Y}, \mathrm{Zr}, \mathrm{Nb}, \mathrm{Mo}, \mathrm{Cs}, \mathrm{Ba}, \mathrm{Hf}, \mathrm{Ta}$, $\mathrm{Pb}$, Th and $\mathrm{U}$ and REE in the Laser Ablation Inductively Coupled Plasma Mass Spectrometry (LA-ICP-MS) at the Institut für Mineralogie, Universität Würzburg in Germany. In this analysis of trace elements, two crystals of plagioclase and two crystals of clinopyroxene were collected from each sample. The laser ablation microprobe was a New Wave (Merchantek) 266 LUV. A Quadrupole MS Agilent 7500i, plasma power $1250 \mathrm{~W}$, carrier gas (Ar) $1.3 \mathrm{~L} / \mathrm{min}$, plasma gas (Ar) $14.9 \mathrm{~L} / \mathrm{min}$ and auxiliary gas (Ar) $0.9 \mathrm{~L} / \mathrm{min}$.
Ablations were performed in single spots with $50 \mu \mathrm{m}$ diameter, repetition rate $10 \mathrm{~Hz}$, laser energy $=$ $0.87^{-1} .10 \mathrm{~mJ}$, laser density $44-53 \mathrm{~J} / \mathrm{cm}^{2}$. Time-resolved analyses were obtained on the maximum peak $18 \mathrm{~s}$, measuring the instrumental + gas background and $22 \mathrm{~s}$ measuring the mineral ( $0 \mathrm{~s}$ on delay) for each analysis.

External calibration via NIST 612, 50 ppm glass-standard, values after Pearce et al. (1997). Other standard used for reproducibility and accuracy: NIST 614, and another chip NIST 612 glass-standard. Internal standard is $\mathrm{SiO}_{2}$ for EPMA measurements. The result was calculated via GLITTER version 3.0 Online Interactive Data Reduction for the LA-ICP-MS, Macquarie Research Ltd. 2000.

The partition coefficient is used for calculating the distribution of trace elements found between the mineral and a melt. It is defined by: $\mathrm{K}_{\mathrm{Di}}$ mineral $=\mathrm{C}_{\mathrm{i}}$ mineral $/$ $\mathrm{C}_{\mathrm{i}}{ }^{\text {melt }}$ where $\mathrm{K}_{\mathrm{D}}$ is the partition coefficient, and $\mathrm{C}$ is the concentration of element $i$ in ppm or wt.\%.

The backscattering images (BSE) by EPMA were used for selecting the best places for analyses of trace elements and REE. Internal zonation was characterized by differences in composition of these elements according to the growth of the plagioclase and clinopyroxene crystals.

\section{PETROGRAPHY AND GEOCHEMISTRY The}

sills were macroscopically analyzed and displayed a color range from light gray to dark gray, aphanitic finegrained (P6220B) to phaneritic coarse-grained. Rocks are holocrystalline $(75-99 \%)$ with primary mineralogy which includes phenocrysts and microphenocrysts of plagioclase, clinopyroxene, opaque minerals and apatite as accessory. The secondary mineralogy comprehends clay minerals and carbonate, presence of alteration products of groundmass replaced by brown clay and Fe oxyhydroxide. The sills from Rio Grande do Sul comprise grains of chalcopyrite (WW223A) and native copper (P6220B), both reaching the maximum size of $0.3 \mathrm{~mm}$. The sills from the other states comprise grains of chalcopyrite and pyrite, sizes ranging from 0.7 up to $2.7 \mathrm{~mm}$. Outside Rio Grande do Sul, the Fe-Ti oxides present larger size, as well as the grains of plagioclase, reaching up to $10.2 \mathrm{~mm}$ and of clinopyroxene, up to $10 \mathrm{~mm}$. The estimated modal distribution of minerals for the sills from Rio Grande do Sul state is: plagioclase $7.5 \sim 26 \%$, clinopyroxene $30 \sim 28 \%$, Fe-Ti oxides $2 \sim 6 \%$ and groundmass $40 \sim 60 \%$ to WW223A and P6220B, respectively. The estimated modal for the sills from Paraná is: plagioclase $30 \sim 42 \%$, clinopyroxene $23 \sim 30 \%$, Fe-Ti oxides $4 \sim 9 \%$ and groundmass $20 \sim 37 \%$. The modal for sills from São Paulo is: plagioclase 18 48\%, clinopyroxene $20 \sim 28 \%$, Fe-Ti oxides $3.5 \sim 15 \%$ and groundmass $8 \sim 41 \%$. The estimated modal for sills from Goiás is: plagioclase $50 \sim 51 \%$, clinopyroxene $23 \sim 36.5 \%$, Fe-Ti oxides 7 15\% and groundmass 1 20\%.

Backscattered electrons images (BSE) of two clinopyroxene samples (Figs. 2A and 2B) and two plagioclase samples (Figs. 2C and 2D) show the analyzed 
spots (laser ablation craters in A). The red circles indicate the area of laser ablation analyses and small filled circles show EPMA analyses. Clinopyroxene and plagioclase display chemical zoning in all crystals examined, but this zoning is either faded or banded in BSE images. In the BSE of clinopyroxene crystals (Fig. 2A), we can see a brighter rim and a diminishing intensity towards its core, identifying Fe increase from the core towards the rim, $\mathrm{Ca}$ and $\mathrm{Mg}$ decreasing concentration from core towards the rim. Thus we classify the clinopyroxene core as a high temperature augite and rim as being pigeonite of lower temperature (Fig. 2B). Figure 2D shows plagioclase oscillatory zonations identified in some of the studied plagioclase samples. The zonations vary according to their chemical composition, core is $\mathrm{Ca}$ rich and darker rim is $\mathrm{Na}$ rich. The rim portions display higher $\mathrm{Si}, \mathrm{Na}$ and $\mathrm{K}$ concentrations and lower $\mathrm{Al}$ and $\mathrm{Ca}$ concentrations. In most of the samples, plagioclase presents $\mathrm{Ca}$ concentration at the core higher than at rim identifying a normal crystallization from high to low temperature.

Bulk rock analyses (Tab. 1) led to chemical classification of the studied rock into basalt and basaltic andesite based on alkali-silica diagram, Zanettin (1984). $\mathrm{Na}_{2} \mathrm{O}+\mathrm{K}_{2} \mathrm{O}$ versus $\mathrm{SiO}_{2}$ wt.\%. $\mathrm{SiO}_{2}$ concentrations varying from 48.6 to $54.7 \mathrm{wt} \%$ and concentrations of $\mathrm{Na}_{2} \mathrm{O}+\mathrm{K}_{2} \mathrm{O}$ between 3 to $5 \mathrm{wt} . \%$. Using as well the major elements $\mathrm{A}=\left(\mathrm{Na}_{2} \mathrm{O}+\mathrm{K}_{2} \mathrm{O}\right), \mathrm{F}=(\mathrm{FeO})$ and $\mathrm{M}=$ $(\mathrm{MgO})$ in wt.\% as shown in the AFM diagram (Irvine \& Baragar 1971), all samples plot in tholeiitic magmas.

The EPMA analyses of clinopyroxene and plagioclase crystals were done on the same areas where LA-ICP-MS analyses were carried out. We also performed analyses showing lines along minerals highlighting the chemical differences in core, intermediate and rim zones (Fig. 2).

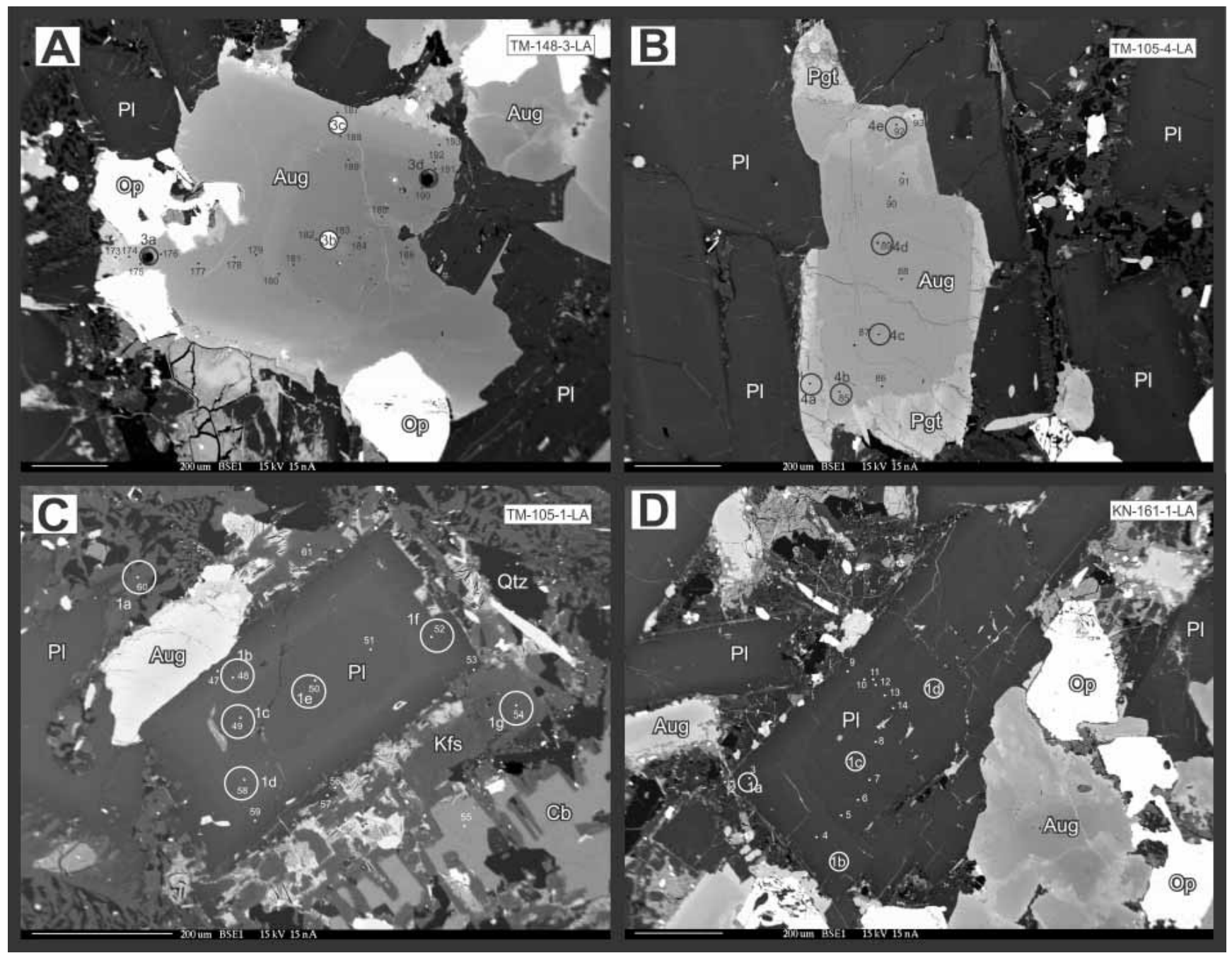

Figure 2 - Backscattered electron images of clinopyroxene (A-) and plagioclase (C-D) analyzed in this study. Open circles show LA-ICP-MS analyses locations. The filled circles show EPMA analyses locations. (A) showing laser ablation craters. Recommendations by the IUGS Subcommission on the Systematics of Metamorphic Rocks: $P l=$ plagioclase; $Q t z=$ quartz; $K f_{s}=$ feldspar-K; Aug = augite; Pgt = pigeonite; $O p=$ opaque minerals and $\mathrm{Cb}$ = carbonate minerals. 
Table 1 - Bulk rock chemical composition of major (wt.\%) and trace elements (ppm) data for sills (basalt and basaltic andesite) in Serra Geral Formation, Paraná Basin, Brazil. Blank = not analyzed.

\begin{tabular}{|c|c|c|c|c|c|c|c|c|c|c|c|c|}
\hline \multirow{2}{*}{$\begin{array}{l}\text { State } \\
\text { Sample }\end{array}$} & \multicolumn{2}{|c|}{ Rio Grande do Sul } & \multicolumn{4}{|c|}{ São Paulo } & \multicolumn{4}{|c|}{ Paraná } & \multicolumn{2}{|c|}{ Goiás } \\
\hline & WW223A & P6220B & TM139 & KN141 & TM134 & TM148 & KN161 & KN164 & KN169 & KN171 & TM102 & TM105 \\
\hline Type & \multicolumn{3}{|c|}{ Basaltic andesite } & & & & & Basalt & & & & \\
\hline $\mathrm{SiO}_{2}$ & 54.76 & 52.59 & 54.28 & 50.02 & 50.93 & 51.16 & 51.96 & 51.35 & 52.06 & 51.67 & 48.86 & 50.18 \\
\hline $\mathrm{TiO}_{2}$ & 1.92 & 1.25 & 2.716 & 2.570 & 3.96 & 3.03 & 2.15 & 1.72 & 2.15 & 2.59 & 2.96 & 3.96 \\
\hline $\mathrm{Al}_{2} \mathrm{O}_{3}$ & 12.29 & 13.22 & 12.31 & 13.00 & 12.47 & 13.37 & 13.09 & 13.16 & 12.81 & 12.85 & 14.02 & 12.01 \\
\hline $\mathrm{Fe}_{2} \mathrm{O}_{3}$ & 14.99 & 13.49 & 13.47 & 15.05 & 14.80 & 13.37 & 14.81 & 13.35 & 15.22 & 15.14 & 14.32 & 16.28 \\
\hline $\mathrm{MnO}$ & 0.19 & 0.18 & 0.18 & 0.21 & 0.17 & 0.20 & 0.21 & 0.20 & 0.22 & 0.21 & 0.18 & 0.21 \\
\hline $\mathrm{MgO}$ & 3.32 & 5.25 & 2.63 & 5.19 & 4.23 & 3.06 & 4.62 & 6.27 & 4.62 & 4.60 & 5.36 & 3.81 \\
\hline $\mathrm{CaO}$ & 7.38 & 8.63 & 6.57 & 9.44 & 7.89 & 7.22 & 8.81 & 10.12 & 8.71 & 8.88 & 9.82 & 7.80 \\
\hline $\mathrm{Na}_{2} \mathrm{O}$ & 2.72 & 2.21 & 2.83 & 2.36 & 2.56 & 3.28 & 2.54 & 2.23 & 2.47 & 2.43 & 2.48 & 2.66 \\
\hline $\mathrm{K}_{2} \mathrm{O}$ & 1.15 & 1.53 & 2.06 & 1.07 & 1.44 & 1.80 & 0.99 & 0.83 & 0.99 & 0.95 & 0.86 & 1.42 \\
\hline $\mathrm{P}_{2} \mathrm{O}_{5}$ & 0.19 & 0.15 & 0.66 & 0.34 & 0.43 & 0.65 & 0.28 & 0.20 & 0.26 & 0.31 & 0.30 & 0.48 \\
\hline LOI & 0.9 & 1.30 & 2.26 & 1.13 & 1.39 & 1.37 & 0.59 & 0.51 & 0.26 & 0.56 & 0.62 & 1.31 \\
\hline Total & 99.82 & 99.81 & 99.96 & 100.37 & 100.27 & 98.52 & 100.04 & 99.95 & 99.78 & 100.20 & 99.80 & 100.12 \\
\hline $\mathrm{FeO}$ & 13.34 & 12.00 & 11.98 & 13.39 & 13.17 & 11.89 & 13.18 & 11.88 & 13.54 & 13.47 & 12.74 & 14.48 \\
\hline $\mathrm{Sc}$ & 39 & 42 & 23 & 37 & 33 & 24 & 39 & 42 & 41 & 41 & 33 & 32 \\
\hline $\mathrm{Ti}$ & 11520 & 7500 & 16296 & 15420 & 23772 & 18216 & 12918 & 10356 & 12930 & 15558 & 17772 & 23760 \\
\hline $\mathrm{V}$ & 550 & 399 & 228 & 481 & 461 & 238 & 377 & 378 & 409 & 420 & 581 & 445 \\
\hline $\mathrm{Cr}$ & & & $<20$ & 95 & 24 & $<20$ & 51 & 131 & 51 & 47 & 106 & $<20$ \\
\hline $\mathrm{Co}$ & 47.8 & 50.2 & 31 & 49 & 43 & 31 & 48 & 40 & 43 & 42 & 48 & 41 \\
\hline $\mathrm{Ni}$ & 6.1 & 14.9 & $<1$ & 34 & 39 & $<1$ & 20 & 49 & 18 & 16 & 55 & $<1$ \\
\hline $\mathrm{Cu}$ & 140 & 203 & 157 & 202 & 136 & 16 & 208 & 167 & 197 & 229 & 102 & 262 \\
\hline $\mathrm{Zn}$ & 51 & 39 & 109 & 99 & 89 & 99 & 102 & 91 & 102 & 105 & 93 & 125 \\
\hline $\mathrm{Rb}$ & 61.7 & 45.3 & 42 & 23 & 30 & 38 & 25 & 17 & 24 & 21 & 17 & 29 \\
\hline $\mathrm{Sr}$ & 228 & 306 & 485 & 371 & 434 & 462 & 302 & 245 & 234 & 230 & 480 & 460 \\
\hline $\mathrm{Y}$ & 32.4 & 24.6 & 53.4 & 34.6 & 39.3 & 43.6 & 36.8 & 30.5 & 40.0 & 45.0 & 25.8 & 41.4 \\
\hline $\mathrm{Zr}$ & 179 & 119 & 360 & 176 & 249 & 268 & 174 & 137 & 187 & 206 & 151 & 260 \\
\hline $\mathrm{Nb}$ & 12.5 & 8.1 & 31.3 & 18.9 & 23.6 & 29.9 & 15.5 & 11.2 & 17.0 & 16.6 & 23.0 & 26.8 \\
\hline Mo & 1.6 & 0.9 & $<2$ & $<2$ & $<2$ & $<2$ & $<2$ & $<2$ & $<2$ & $<2$ & $<2$ & $<2$ \\
\hline Cs & 2.3 & 1.1 & 0.5 & 0.2 & 0.3 & 0.4 & 0.2 & 0.3 & 0.5 & 0.2 & 0.2 & 0.3 \\
\hline $\mathrm{Ba}$ & 454 & 281 & 668 & 377 & 481 & 570 & 356 & 265 & 306 & 272 & 329 & 464 \\
\hline $\mathrm{Ga}$ & 19 & 18 & 28 & 24 & 25 & 26 & 24 & 18 & 22 & 22 & 25 & 26 \\
\hline $\mathrm{La}$ & 23.50 & 14.00 & 52.54 & 23.98 & 33.42 & 43.47 & 22.13 & 16.85 & 23.92 & 23.88 & 24.98 & 38.34 \\
\hline $\mathrm{Ce}$ & 53.00 & 32.70 & 110.90 & 52.77 & 71.85 & 90.09 & 48.40 & 36.46 & 51.23 & 52.87 & 53.49 & 81.02 \\
\hline $\mathrm{Pr}$ & 6.87 & 4.31 & 14.03 & 6.30 & 9.06 & 11.34 & 5.84 & 4.43 & 6.17 & 6.59 & 6.64 & 10.07 \\
\hline $\mathrm{Nd}$ & 28.80 & 18.60 & 60.48 & 27.50 & 40.02 & 46.94 & 25.24 & 19.39 & 26.69 & 29.59 & 28.99 & 43.52 \\
\hline $\mathrm{Sm}$ & 6.32 & 4.36 & 12.80 & 6.05 & 9.05 & 9.86 & 5.73 & 4.66 & 6.42 & 7.29 & 6.43 & 9.54 \\
\hline $\mathrm{Eu}$ & 1.55 & 1.22 & 3.95 & 2.07 & 2.87 & 3.26 & 1.94 & 1.61 & 2.08 & 2.39 & 2.26 & 3.19 \\
\hline $\mathrm{Gd}$ & 6.25 & 4.42 & 12.44 & 6.37 & 8.76 & 9.89 & 6.04 & 5.23 & 7.36 & 8.28 & 6.11 & 9.18 \\
\hline $\mathrm{Tb}$ & 1.1 & 0.8 & 1.9 & 1.0 & 1.3 & 1.5 & 1.0 & 0.9 & 1.2 & 1.4 & 0.9 & 1.4 \\
\hline Dy & 6.09 & 4.63 & 10.09 & 5.85 & 7.50 & 8.20 & 5.91 & 5.47 & 7.19 & 8.22 & 5.16 & 7.83 \\
\hline Ho & 1.13 & 0.87 & 1.85 & 1.17 & 1.40 & 1.52 & 1.15 & 1.06 & 1.44 & 1.62 & 0.94 & 1.45 \\
\hline $\mathrm{Er}$ & 3.20 & 2.46 & 5.29 & 3.44 & 4.15 & 4.40 & 3.58 & 3.29 & 4.41 & 4.94 & 2.78 & 4.29 \\
\hline $\mathrm{Tm}$ & 0.45 & 0.36 & 0.71 & 0.48 & 0.56 & 0.61 & 0.51 & 0.47 & 0.64 & 0.69 & 0.36 & 0.58 \\
\hline $\mathrm{Yb}$ & 3.12 & 2.41 & 4.44 & 3.05 & 3.40 & 3.64 & 3.21 & 2.86 & 3.96 & 4.30 & 2.22 & 3.49 \\
\hline $\mathrm{Lu}$ & 0.44 & 0.34 & 0.58 & 0.42 & 0.47 & 0.50 & 0.46 & 0.41 & 0.55 & 0.62 & 0.30 & 0.47 \\
\hline $\mathrm{Hf}$ & 4.8 & 3.4 & 9.4 & 4.6 & 6.7 & 7.1 & 4.4 & 3.7 & 5.2 & 5.5 & 4.3 & 6.9 \\
\hline $\mathrm{Ta}$ & 0.70 & 0.50 & 2.00 & 1.08 & 1.58 & 1.86 & 0.91 & 0.71 & 1.00 & 1.02 & 1.04 & 1.76 \\
\hline W & 0.4 & 1.1 & $<0.5$ & $<0.5$ & $<0.5$ & $<0.5$ & $<0.5$ & $<0.5$ & $<0.5$ & $<0.5$ & $<0.5$ & $<0.5$ \\
\hline $\mathrm{Pb}$ & 1.4 & 2 & 19 & $<3$ & 23 & $<3$ & 13 & 11 & 5 & 15 & 9 & 13 \\
\hline $\mathrm{Th}$ & 7.70 & 3.90 & 4.57 & 2.67 & 3.10 & 3.66 & 2.43 & 1.89 & 2.57 & 2.26 & 1.96 & 3.38 \\
\hline $\mathrm{U}$ & 1.50 & 1.10 & 0.92 & 0.56 & 0.64 & 0.73 & 0.46 & 0.36 & 0.48 & 0.45 & 0.40 & 0.70 \\
\hline
\end{tabular}


Plagioclase The microanalyses classified plagioclase according to Or-Ab-An diagram (Deer et al. 2003) (Fig. 3 ) in portions which vary from oligoclase to labradorite $\left(\mathrm{An}_{20-68} \mathrm{Ab}_{31-74} \mathrm{Or}_{1-8}\right)$. Table 2 shows the oxides $\mathrm{Si}, \mathrm{Al}$, $\mathrm{Fe}, \mathrm{Mg}, \mathrm{Ca}, \mathrm{Na}, \mathrm{K}$ and $\mathrm{Ti}$ mean values analyzed by EPMA in plagioclase crystals. Plagioclase fractionation of crystals in those samples present the expected results, increasing values from core towards rim for $\mathrm{Si}, \mathrm{K}$ and $\mathrm{Na}$ oxides and decreasing $\mathrm{Al}, \mathrm{Mg}$ and $\mathrm{Ca}$ values from core towards rim. In most samples $\mathrm{FeO}$ and $\mathrm{TiO}_{2}$ wt.\% values have a decrease from core towards rim except WW223A and KN161 samples results which present different values. The plagioclase rims present values of those oxides with large variations related to core and intermediate zones, those variations can be related to the end of mineral crystallization and last stages of chemical/thermal balance of those elements.

The analyses of some major elements by EPMA (Fig. 4) made it possible to identify chemical variations related to crystallization of plagioclase at rim, intermediate and core zones. Negative correlations of $\mathrm{CaO}$ and $\mathrm{SiO}_{2}$ show a normal fractioning of $\mathrm{Ca}$ oxides with larger concentrations in the core portions of the mineral and silica at the rim zones (Figs. 4a, $4 \mathrm{~d}$ and $4 \mathrm{~g}$ ). The binary diagrams show a positive correlation between $\mathrm{TiO}_{2}$ and $\mathrm{Al}_{2} \mathrm{O}_{3}$ in plagioclase, high concentrations of $\mathrm{TiO}_{2}$ wt. $\%$ and $\mathrm{Al}_{2} \mathrm{O}_{3}$ wt. $\%$ at the core and low concentrations at the rim (Figs. 4b, $4 \mathrm{e}$ and $4 \mathrm{~h}$ ). Figures $4 \mathrm{c}, 4 \mathrm{f}$ and $4 \mathrm{i}$ show lower values for $\mathrm{FeO}$ wt.\% and $\mathrm{An} \%$ at rim and increase of concentration at the core, identifying positive correlations of those elements.

Figure 5 shows binary diagrams of $\mathrm{An} \%$ versus $\mathrm{FeO}, \mathrm{TiO}_{2}, \mathrm{~K}_{2} \mathrm{O}$ and $\mathrm{Al}_{2} \mathrm{O}_{3}$ wt.\%. The plagioclase fractionation is considered normal, according to the $\mathrm{An} \%$ with $\mathrm{Al}_{2} \mathrm{O}_{3}$ and $\mathrm{FeO}$ wt.\% positive correlations (Figs. 5a and 5d). In relation to Na negative correlations, we highlight the low values $\mathrm{TiO}_{2}$ for the plagioclase sills collected in Rio Grande do Sul compared to those in other states (Fig. 5c).

Clinopyroxene The microanalyses of clinopyroxene in sills classified the mineral into two chemical types: high$\mathrm{Ca}$ (augite) and low-Ca (pigeonite), according to table 3. According to quadrilateral diagram from Morimoto (1988) and isotherms modified from Lindsley (1983) to $\mathrm{P}=1 \mathrm{kbar}$, the clinopyroxene core, intermediate and rim zones results were plotted (Fig. 6). Most clinopyroxene has pigeonite rims of lower temperatures $\left(\sim 700^{\circ} \mathrm{C}\right.$ to $950^{\circ} \mathrm{C}$ ), however, some clinopyroxene have augite rims, of temperature equivalent to those of pigeonite rims, showing that differences between the two chemical terms do not influence temperature in the several sills we studied. The core of the respective clinopyroxenes (augite and pigeonite) have the same behavior, different nuclei composition but similar temperatures $\left(\sim 1150^{\circ} \mathrm{C}\right.$ to $1100^{\circ} \mathrm{C}$ ), are very well represented by sills samples from Paraná state. No pigeonite nuclei were identified in sills samples from São Paulo, only low temperature rims.

Figure 7 shows a positive correlation between $\mathrm{Al}_{2} \mathrm{O}_{3}$ wt. $\%$ and $\mathrm{Wo} \%, \mathrm{TiO}_{2}$ wt. $\%$ and $\mathrm{Al}_{2} \mathrm{O}_{3}$ wt.\% and negative correlation between $\mathrm{MgO}$ wt.\% and Fs\% from clinopyroxene microanalyses. According to Wo\% values, we can separate clinopyroxene into two groups (augite $>22 \mathrm{Wo} \%$ and pigeonite $<22 \mathrm{Wo} \%$ ) (Figs. $7 \mathrm{a}, 7 \mathrm{~d}$ and $7 \mathrm{~g}$ ). Values above 1.0 wt. $\% \mathrm{Al}_{2} \mathrm{O}_{3}$

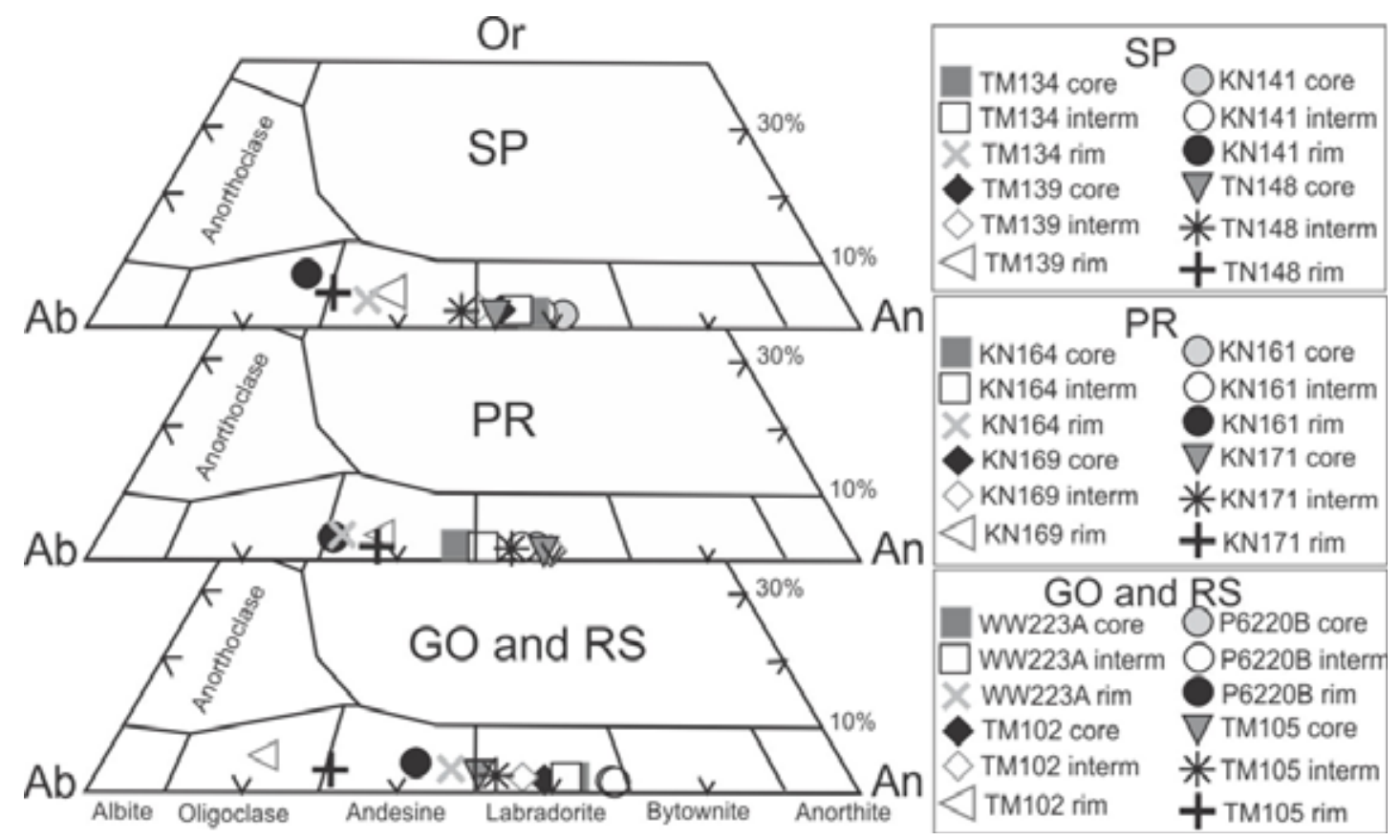

Figure 3 - Plagioclase composition ternary diagram (core to rim) Deer et al. (2003) from São Paulo (SP), Paraná $(P R)$, Goiás (GO) and Rio Grande do Sul (RS). Aug = augite and pgt = pigeonite. 


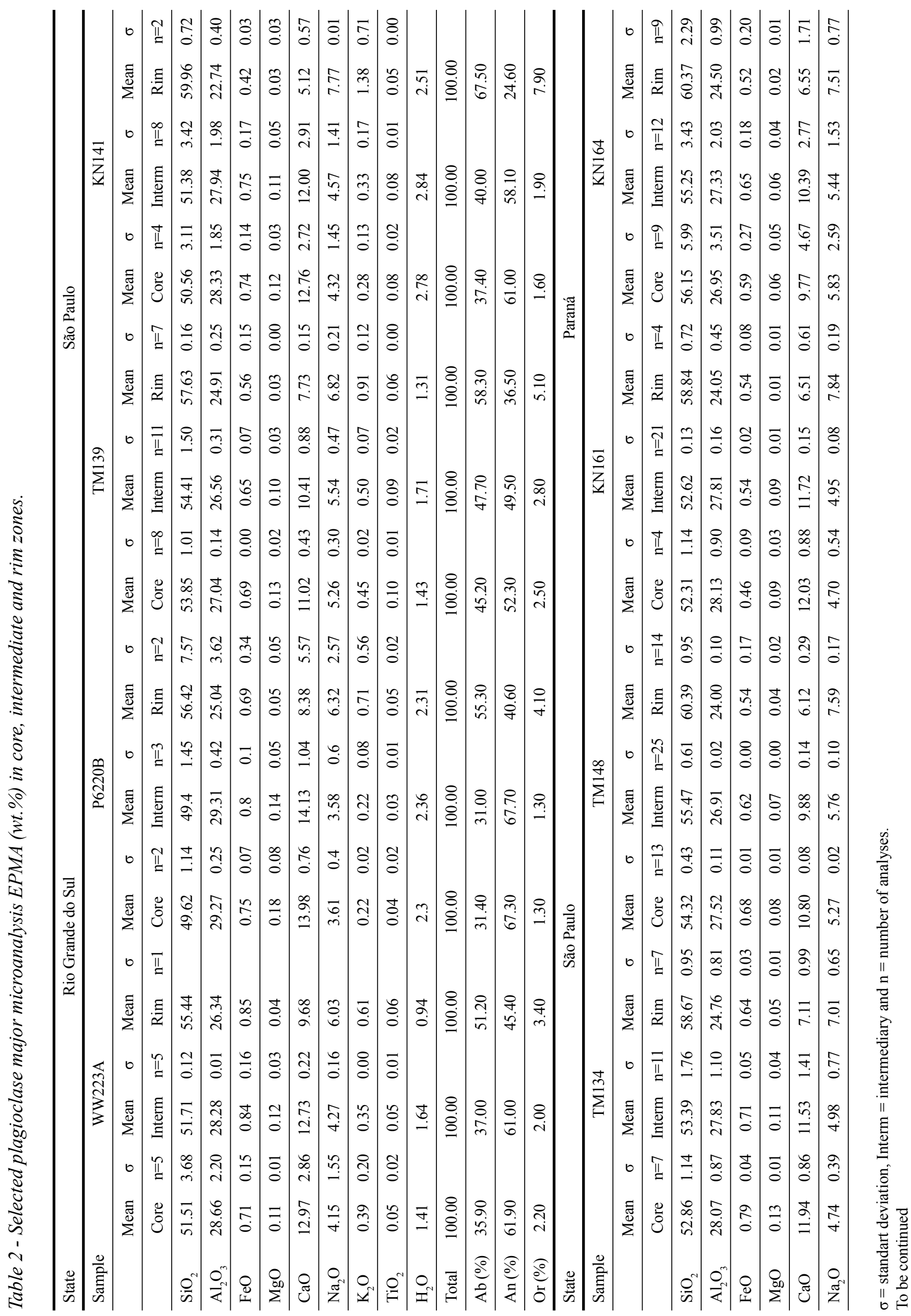




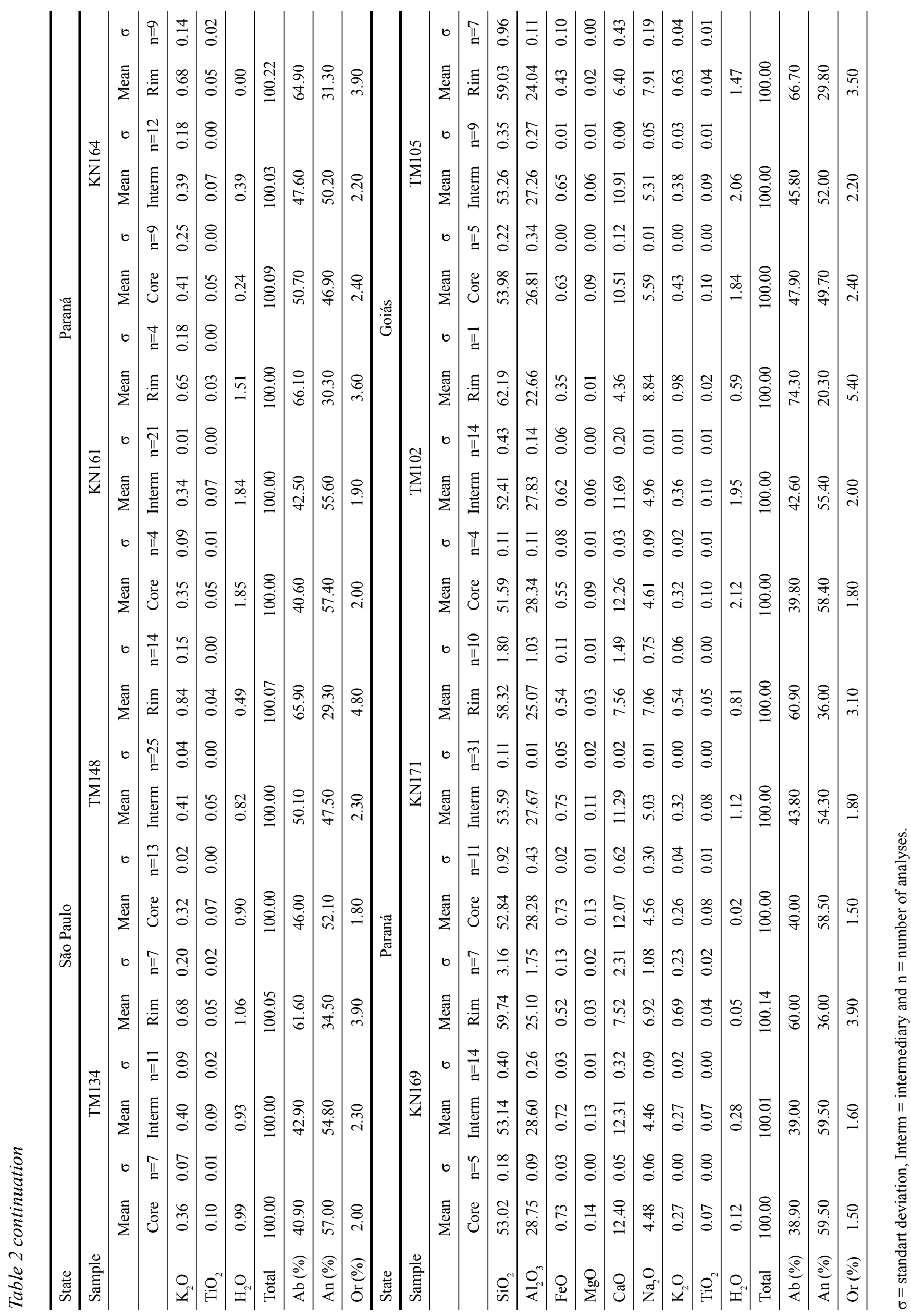


classify the core and intermediate portions of the mineral, either augite or pigeonite. Figure $7 \mathrm{~b}$ shows the values below 1 for $\mathrm{TiO}$ and $\mathrm{Al}_{2} \mathrm{O}_{3}$ wt.\% in rims of augite and pigeonite in Goiás and Rio Grande do Sul states. Negative correlation is identified in diagrams of $\mathrm{MgO}$ wt.\% versus $\mathrm{Fs} \%$ in figures $7 \mathrm{c}, 7 \mathrm{f}$ and 7 i. Figure $7 \mathrm{c}$ shows that higher $\mathrm{MgO}$ wt.\% values are related to clinopyroxene nuclei and pigeonite nuclei to higher values. In figures $7 \mathrm{c}$ and $7 \mathrm{f}$, there are two trends related to chemical compositions of core, intermediate and rim zones for augite and pigeonite. Figure 7i of São Paulo state does not represent two trends, but presents the highest Fs\% values for augite and pigeonite.

The EPMAmean microanalyses of clinopyroxene crystals (Fig. 8) shows $\mathrm{TiO}_{2}$ and $\mathrm{Al}_{2} \mathrm{O}_{3}$ wt.\% variations versus $\mathrm{En}, \mathrm{Fs}$ and $\mathrm{Wo} \%$ contents for samples from
Rio Grande do Sul, Paraná, São Paulo and Goiás. The highest concentration values for $\mathrm{TiO}_{2}$ and $\mathrm{Al}_{2} \mathrm{O}_{3}$ wt.\% found in clinopyroxene are in São Paulo, followed by Goiás, Paraná and the lowest values are in Rio Grande do Sul. Positive correlations are found in $\mathrm{Al}_{2} \mathrm{O}_{3}$ wt.\% versus En and $\mathrm{Wo} \%$ diagram, figures $8 \mathrm{~b}$ and $8 \mathrm{f}$ and $\mathrm{TiO}_{2}$ wt. $\%$ versus $\mathrm{Wo} \%$, figures $8 \mathrm{a}$ and $8 \mathrm{~b}$. Lowest $\mathrm{TiO}_{2}$ wt. $\%$ concentrations in clinopyroxene are evidence of low concentration of that oxide in other minerals as plagioclase and Ti-magnetite and ilmenite.

RESULTS - PARTITION COEFFICIENTS The partition coefficients of trace elements in plagioclase and clinopyroxene crystals in sills of Serra Geral Formation in Paraná Basin describe the mineral $/$ melt preference. The total rock analyses made it possible to
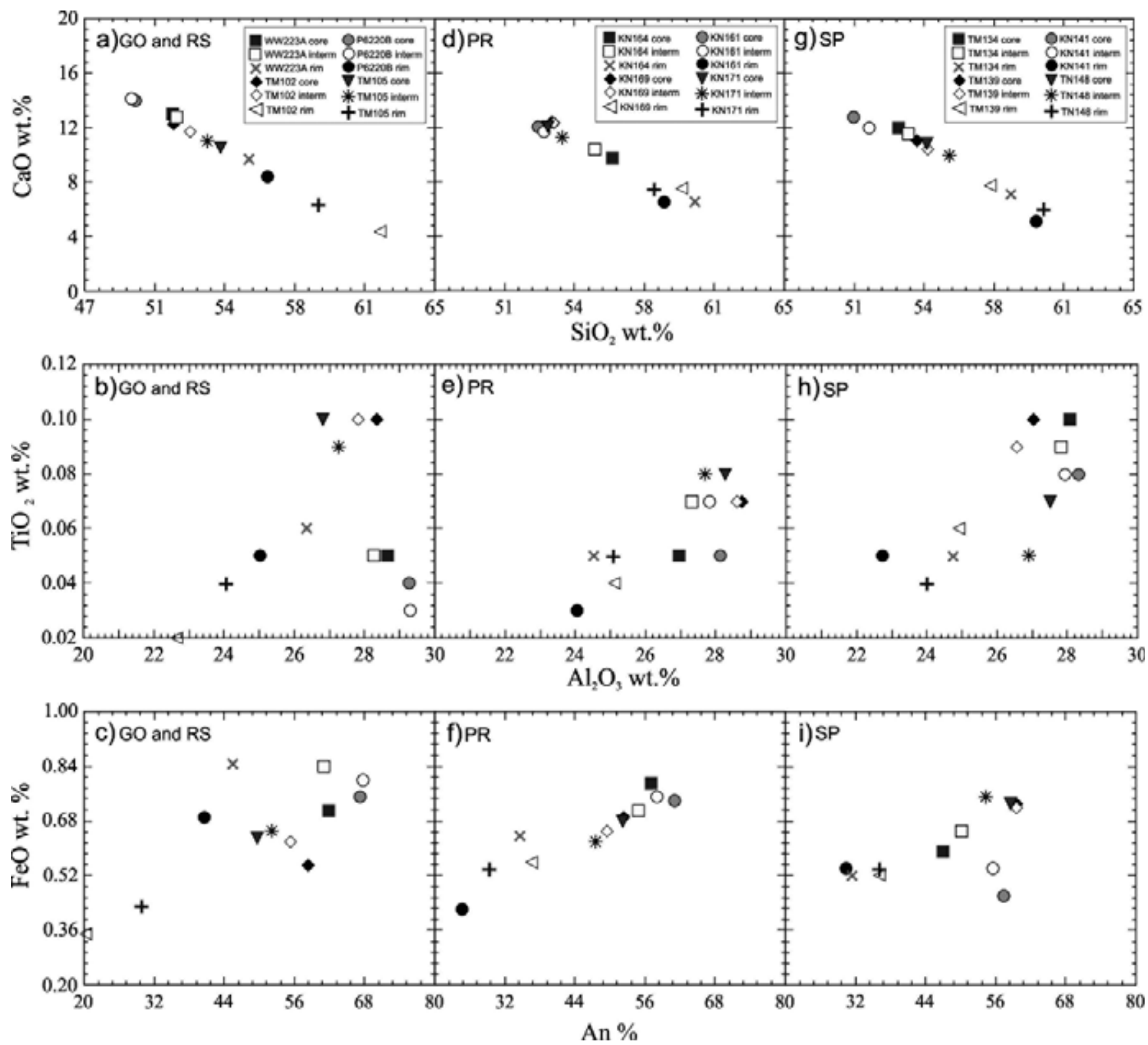

Figure 4 - $\mathrm{CaO}$ wt.\% versus $\mathrm{SiO}_{2}$ wt. \%, $\mathrm{TiO}_{2}$ wt.\% versus $\mathrm{Al}_{2} \mathrm{O}_{3}$ wt. \% and $\mathrm{FeO}$ wt.\% versus $\mathrm{An} \%$ for plagioclase average microanalyses from EPMA in core, intermediate and rim zones. Aug = augite and pgt $=$ pigeonite. 

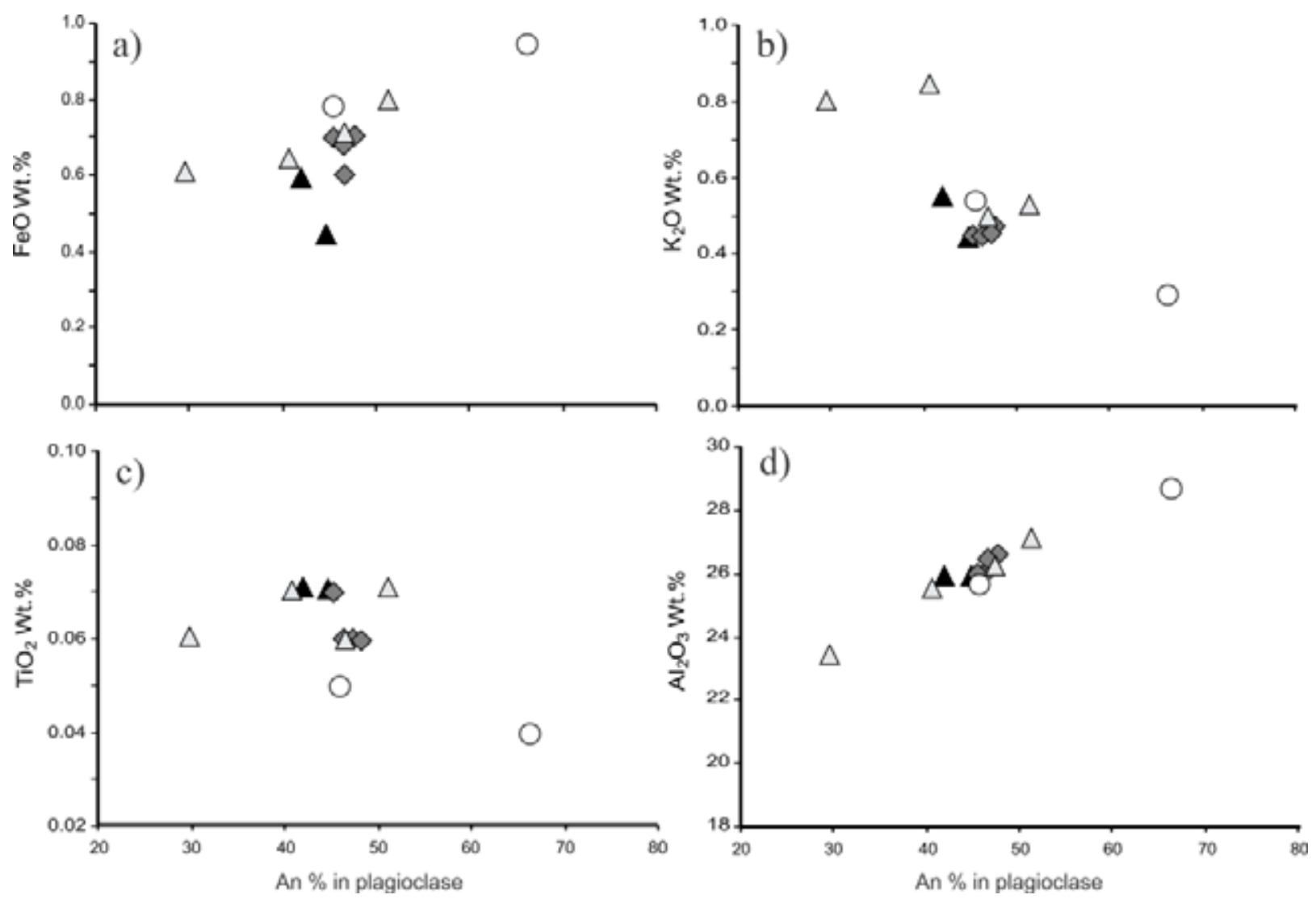

Figure 5 - Binary diagram show the distribution between $\mathrm{FeO}, \mathrm{K}_{2} \mathrm{O}, \mathrm{TiO}_{2}$ and $\mathrm{Al}_{2} \mathrm{O}_{3}$ wt.\% and anorthite content in plagioclase average microanalyses from EPMA in sills of Serra Geral Formation, Paraná Basin. Black triangle: plagioclase from Goiás; gray triangle: São Paulo; gray lozenge: Paraná; white circle: Rio Grande do Sul.

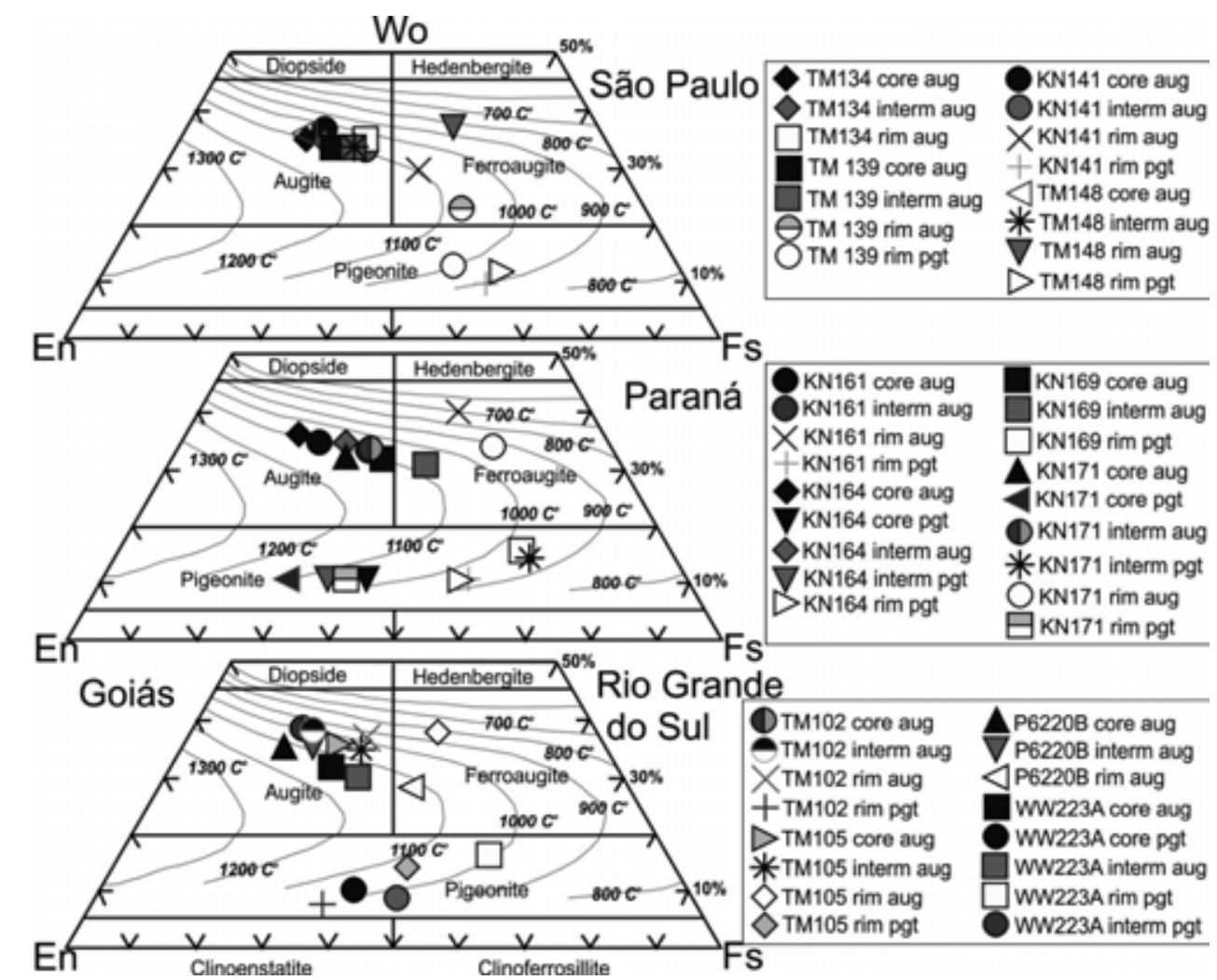

Figure 6 - Pyroxene classification quadrilateral diagram [Ca, $\mathrm{Mg}, \mathrm{Fe}(\mathrm{Fe}+\mathrm{Mn}$ ) in mol\%] from Morimoto (1988). Isotherms modified Lindsley (1983) to $P=1 \mathrm{kbar}$. Aug = augite and pgt = pigeonite. 
Table 3 - Representative major elements EPMA (wt.\%) compositions of clinopyroxene in core, intermediate and rim zones.

\begin{tabular}{|c|c|c|c|c|c|c|c|c|c|c|c|c|c|c|c|c|c|c|}
\hline State & & & & & & & & & io Grand & e do $\mathrm{S}$ & & & & & & & & \\
\hline Sample & & & & & WW2 & $23 \mathrm{~A}$ & & & & & & & & P62 & 20B & & & \\
\hline & Mean & $\sigma$ & Mean & $\sigma$ & Mean & $\sigma$ & Mean & $\sigma$ & Mean & $\sigma$ & Mean & $\sigma$ & Mean & $\sigma$ & Mean & $\sigma$ & Mean & $\sigma$ \\
\hline & $\begin{array}{l}\text { Core } \\
\text { Aug }\end{array}$ & $\mathrm{n}=3$ & $\begin{array}{l}\text { Core } \\
\text { Pgt }\end{array}$ & $\mathrm{n}=2$ & $\begin{array}{l}\text { Interm } \\
\text { Aug }\end{array}$ & $\mathrm{n}=1$ & $\begin{array}{c}\text { Interm } \\
\text { Pgt }\end{array}$ & $\mathrm{n}=4$ & $\begin{array}{c}\text { Rim } \\
\text { Pgt }\end{array}$ & $\mathrm{n}=4$ & $\begin{array}{l}\text { Core } \\
\text { Aug }\end{array}$ & $\mathrm{n}=4$ & $\begin{array}{c}\text { Interm } \\
\text { Aug }\end{array}$ & $\mathrm{n}=5$ & $\begin{array}{l}\text { Rim } \\
\text { Aug }\end{array}$ & $\mathrm{n}=4$ & & \\
\hline $\mathrm{SiO}_{2}$ & 50.12 & 0.31 & 51.51 & 0.46 & 51.01 & & 49.73 & 1.39 & 48.27 & 0.62 & 50.73 & 0.29 & 49.03 & 0.52 & 47.4 & 0.68 & & \\
\hline $\mathrm{Al}_{2} \mathrm{O}_{3}$ & 1.58 & 0.31 & 0.87 & 0.00 & 1.54 & & 0.79 & 0.07 & 0.66 & 0.08 & 1.62 & 0.26 & 1.58 & 0.12 & 1.15 & 0.3 & & \\
\hline $\mathrm{FeO}$ & 15.22 & 1.79 & 24.28 & 0.78 & 18.22 & & 27.60 & 4.39 & 32.94 & 1.22 & 9.71 & 1.51 & 12.35 & 0.54 & 23.13 & 0.65 & & \\
\hline $\mathrm{MnO}$ & 0.34 & 0.02 & 0.50 & 0.07 & 0.37 & & 0.56 & 0.08 & 0.66 & 0.01 & 0.25 & 0.00 & 0.26 & 0.05 & 0.45 & 0.15 & & \\
\hline $\mathrm{MgO}$ & 15.64 & 0.55 & 18.28 & 1.03 & 14.27 & & 15.57 & 3.46 & 9.21 & 0.75 & 17.84 & 1.25 & 15.74 & 0.07 & 11.47 & 0.98 & & \\
\hline $\mathrm{CaO}$ & 15.65 & 1.97 & 4.95 & 0.28 & 14.75 & & 4.03 & 0.22 & 7.48 & 2.14 & 17.89 & 0.03 & 17.38 & 0.78 & 13.45 & 3.98 & & \\
\hline $\mathrm{Na}_{2} \mathrm{O}$ & 0.14 & 0.04 & 0.05 & 0.02 & 0.12 & & 0.04 & 0.02 & 0.07 & 0.04 & 0.18 & 0.04 & 0.55 & 0.47 & 0.18 & 0.02 & & \\
\hline $\mathrm{TiO}_{2}$ & 0.59 & 0.05 & 0.36 & 0.03 & 0.61 & & 0.36 & 0.05 & 0.48 & 0.03 & 0.33 & 0.12 & 0.41 & 0.1 & 0.72 & 0.04 & & \\
\hline $\mathrm{NiO}$ & 0.02 & 0.03 & 0.00 & 0.00 & 0.06 & & 0.02 & 0.02 & 0.01 & 0.00 & 0.03 & 0.00 & 0.02 & 0.01 & 0.02 & 0.03 & & \\
\hline $\mathrm{Cr}_{2} \mathrm{O}_{3}$ & 0.03 & 0.01 & 0.01 & 0.01 & 0.03 & & 0.01 & 0.01 & 0.00 & 0.00 & 0.05 & 0.00 & 0.05 & 0.01 & 0.01 & 0.01 & & \\
\hline $\mathrm{H}_{2} \mathrm{O}$ & 0.65 & & 0.00 & & 0.00 & & 0.46 & & 0.43 & & 1.37 & & 1.73 & & 1.98 & & & \\
\hline Total & 100.00 & & 100.81 & & 101.00 & & 100.07 & & 100.23 & & 100.00 & & 100.00 & & 100.00 & & & \\
\hline $\begin{array}{l}\text { Wo } \\
(\%)\end{array}$ & 31.58 & & 9.95 & & 30.03 & & 8.45 & & 16.08 & & 35.43 & & 35.38 & & 28.13 & & & \\
\hline En (\%) & 43.91 & & 51.14 & & 40.42 & & 45.44 & & 27.54 & & 49.16 & & 44.58 & & 33.37 & & & \\
\hline Fs $(\%)$ & 24.51 & & 38.90 & & 29.55 & & 46.11 & & 56.38 & & 15.40 & & 20.04 & & 38.50 & & & \\
\hline State & & & & & & & & & São P & aulo & & & & & & & & \\
\hline Sample & & & & $\mathrm{TM}$ & 1139 & & & & & & & & KN1 & & & & & \\
\hline & Mean & $\sigma$ & Mean & $\sigma$ & Mean & $\sigma$ & Mean & $\sigma$ & Mean & $\sigma$ & Mean & $\sigma$ & Mean & $\sigma$ & Mean & $\sigma$ & Mean & $\sigma$ \\
\hline & $\begin{array}{l}\text { Core } \\
\text { Aug }\end{array}$ & $\mathrm{n}=8$ & $\begin{array}{c}\text { Interm } \\
\text { Aug }\end{array}$ & $\mathrm{n}=9$ & $\begin{array}{l}\text { Rim } \\
\text { Aug }\end{array}$ & $\mathrm{n}=1$ & $\begin{array}{l}\text { Rim } \\
\text { Pgt }\end{array}$ & $\mathrm{n}=3$ & $\begin{array}{l}\text { Core } \\
\text { Aug }\end{array}$ & $\mathrm{n}=6$ & $\begin{array}{c}\text { Interm } \\
\text { Aug }\end{array}$ & $\mathrm{n}=9$ & $\begin{array}{l}\text { Rim } \\
\text { Aug }\end{array}$ & $\mathrm{n}=1$ & $\begin{array}{c}\text { Rim } \\
\text { Pgt }\end{array}$ & $\mathrm{n}=1$ & & \\
\hline $\mathrm{SiO}_{2}$ & 49.07 & 0.72 & 49.42 & 0.50 & 47.50 & & 48.56 & 0.51 & 49.56 & 0.29 & 49.15 & 0.52 & 47.80 & & 48.39 & & & \\
\hline $\mathrm{Al}_{2} \mathrm{O}_{3}$ & 2.23 & 0.06 & 1.65 & 0.11 & 0.74 & & 0.67 & 0.16 & 1.78 & 0.19 & 1.31 & 0.07 & 0.86 & & 0.23 & & & \\
\hline $\mathrm{FeO}$ & 14.86 & 0.22 & 16.64 & 0.58 & 28.93 & & 31.35 & 2.75 & 13.27 & 0.20 & 17.97 & 0.31 & 23.11 & & 34.32 & & & \\
\hline $\mathrm{MnO}$ & 0.36 & 0.01 & 0.43 & 0.02 & 0.76 & & 0.83 & 0.04 & 0.32 & 0.02 & 0.47 & 0.01 & 0.66 & & 0.95 & & & \\
\hline $\mathrm{MgO}$ & 14.79 & 0.21 & 13.87 & 0.19 & 9.84 & & 11.82 & 1.24 & 14.86 & 0.34 & 13.01 & 0.07 & 10.56 & & 10.35 & & & \\
\hline $\mathrm{CaO}$ & 16.36 & 0.49 & 16.13 & 0.23 & 10.42 & & 5.85 & 1.03 & 17.77 & 0.27 & 15.97 & 0.37 & 14.07 & & 4.46 & & & \\
\hline $\mathrm{Na}_{2} \mathrm{O}$ & 0.23 & 0.03 & 0.22 & 0.01 & 0.13 & & 0.08 & 0.01 & 0.26 & 0.03 & 0.21 & 0.00 & 0.12 & & 0.07 & & & \\
\hline $\mathrm{TiO}_{2}$ & 1.29 & 0.07 & 0.97 & 0.06 & 0.53 & & 0.49 & 0.10 & 1.02 & 0.12 & 0.85 & 0.03 & 0.65 & & 0.35 & & & \\
\hline $\mathrm{NiO}$ & 0.02 & 0.01 & 0.01 & 0.02 & 0.00 & & 0.01 & 0.02 & 0.01 & 0.00 & 0.01 & 0.01 & 0.04 & & 0.03 & & & \\
\hline $\mathrm{Cr}_{2} \mathrm{O}_{3}$ & 0.01 & 0.00 & 0.01 & 0.01 & 0.00 & & 0.00 & 0.01 & 0.00 & 0.00 & 0.01 & 0.00 & 0.01 & & 0.00 & & & \\
\hline $\mathrm{H}_{2} \mathrm{O}$ & 0.82 & & 0.70 & & 1.12 & & 0.43 & & 1.14 & & 1.04 & & 2.10 & & 0.84 & & & \\
\hline Total & 100.03 & & 100.07 & & 100.00 & & 100.09 & & 100.00 & & 100.00 & & 100.00 & & 100.00 & & & \\
\hline $\begin{array}{l}\text { Wo } \\
(\%)\end{array}$ & 33.51 & & 33.08 & & 22.03 & & 12.34 & & 36.22 & & 32.95 & & 29.73 & & 9.61 & & & \\
\hline En (\%) & 42.15 & & 39.58 & & 28.95 & & 34.68 & & 42.15 & & 37.35 & & 31.05 & & 31.04 & & & \\
\hline Fs $(\%)$ & 24.34 & & 27.34 & & 49.02 & & 52.98 & & 21.63 & & 29.71 & & 39.22 & & 59.35 & & & \\
\hline
\end{tabular}

$\sigma=$ standart deviation, Interm $=$ intermediary, $\mathrm{Aug}=$ augite and Pgt $=$ pigeonite and $\mathrm{n}=$ number of analyses.

To be continued 
Table 3 continuation

\begin{tabular}{|c|c|c|c|c|c|c|c|c|c|c|c|c|c|c|c|c|c|c|}
\hline \multirow{3}{*}{$\frac{\text { State }}{\text { Sample }}$} & \multicolumn{18}{|c|}{ Paraná } \\
\hline & \multicolumn{6}{|c|}{ KN169 } & \multicolumn{12}{|c|}{ KN171 } \\
\hline & Mean & $\sigma$ & Mean & $\sigma$ & Mean & $\sigma$ & Mean & $\sigma$ & Mean & $\sigma$ & Mean & $\sigma$ & Mean & $\sigma$ & Mean & $\sigma$ & Mean & $\sigma$ \\
\hline & $\begin{array}{l}\text { Core } \\
\text { Aug }\end{array}$ & $n=14$ & $\begin{array}{l}\text { Interm } \\
\text { Aug }\end{array}$ & $\mathrm{n}=14$ & $\begin{array}{l}\text { Rim } \\
\text { Pgt }\end{array}$ & $\mathrm{n}=10$ & $\begin{array}{l}\text { Core } \\
\text { Aug }\end{array}$ & $\mathrm{n}=10$ & $\begin{array}{l}\text { Core } \\
\text { Pgt }\end{array}$ & $\mathrm{n}=3$ & $\begin{array}{c}\text { Interm } \\
\text { Aug }\end{array}$ & $\mathrm{n}=20$ & $\begin{array}{c}\text { Interm } \\
\text { Pgt }\end{array}$ & $\mathrm{n}=2$ & $\begin{array}{l}\text { Rim } \\
\text { Aug }\end{array}$ & $\mathrm{n}=3$ & $\begin{array}{l}\text { Rim } \\
\text { Pgt }\end{array}$ & $\mathrm{n}=4$ \\
\hline $\mathrm{SiO}_{2}$ & 50.35 & 0.29 & 49.61 & 0.38 & 48.01 & 0.44 & 50.30 & 0.35 & 52.33 & 0.45 & 49.87 & 0.47 & 51.20 & 0.09 & 48.49 & 0.80 & 47.51 & 0.23 \\
\hline $\mathrm{Al}_{2} \mathrm{O}_{3}$ & 1.16 & 0.09 & 1.05 & 0.10 & 0.57 & 0.11 & 1.51 & 0.21 & 0.95 & 0.24 & 1.40 & 0.26 & 0.81 & 0.07 & 0.48 & 0.25 & 0.50 & 0.16 \\
\hline $\mathrm{FeO}$ & 19.59 & 1.12 & 23.42 & 2.17 & 35.57 & 2.84 & 16.35 & 0.61 & 18.04 & 0.74 & 17.51 & 2.43 & 23.28 & 0.25 & 27.70 & 1.13 & 35.90 & 2.49 \\
\hline $\mathrm{MnO}$ & 0.44 & 0.03 & 0.55 & 0.04 & 0.78 & 0.06 & 0.38 & 0.04 & 0.39 & 0.03 & 0.41 & 0.08 & 0.48 & 0.02 & 0.62 & 0.03 & 0.78 & 0.05 \\
\hline $\mathrm{MgO}$ & 12.63 & 0.43 & 10.37 & 1.22 & 7.65 & 1.26 & 14.35 & 0.35 & 22.16 & 0.67 & 13.11 & 1.45 & 18.55 & 0.33 & 6.21 & 0.18 & 7.50 & 0.82 \\
\hline $\mathrm{CaO}$ & 15.03 & 0.88 & 14.35 & 1.02 & 6.95 & 1.45 & 15.54 & 0.60 & 5.20 & 1.40 & 16.00 & 0.97 & 4.98 & 0.31 & 15.52 & 1.01 & 6.77 & 1.84 \\
\hline $\mathrm{Na}_{2} \mathrm{O}$ & 0.16 & 0.03 & 0.16 & 0.04 & 0.07 & 0.03 & 0.20 & 0.04 & 0.05 & 0.04 & 0.19 & 0.04 & 0.06 & 0.04 & 0.15 & 0.06 & 0.08 & 0.03 \\
\hline $\mathrm{TiO}_{2}$ & 0.74 & 0.06 & 0.71 & 0.07 & 0.49 & 0.06 & 0.89 & 0.12 & 0.40 & 0.06 & 0.89 & 0.10 & 0.48 & 0.01 & 0.36 & 0.21 & 0.50 & 0.05 \\
\hline $\mathrm{NiO}$ & 0.02 & 0.02 & 0.01 & 0.02 & 0.02 & 0.02 & 0.01 & 0.02 & 0.04 & 0.01 & 0.02 & 0.03 & 0.03 & 0.02 & 0.01 & 0.01 & 0.01 & 0.01 \\
\hline $\mathrm{Cr}_{2} \mathrm{O}_{3}$ & 0.01 & 0.01 & 0.00 & 0.00 & 0.01 & 0.01 & 0.01 & 0.01 & 0.05 & 0.01 & 0.01 & 0.01 & 0.01 & 0.01 & 0.00 & 0.01 & 0.00 & 0.00 \\
\hline $\mathrm{H}_{2} \mathrm{O}$ & 0.05 & & 0.02 & & 0.05 & & 0.46 & & 0.47 & & 0.60 & & 0.12 & & 0.43 & & 0.45 & \\
\hline Total & 100.19 & & 100.25 & & 100.16 & & 100.00 & & 100.10 & & 100.01 & & 100.00 & & 100.00 & & 100.00 & \\
\hline $\begin{array}{l}\text { Wo } \\
(\%)\end{array}$ & 31.16 & & 30.21 & & 15.12 & & 32.00 & & 10.31 & & 33.17 & & 10.09 & & 33.54 & & 14.77 & \\
\hline En $(\%)$ & 36.43 & & 30.38 & & 23.15 & & 41.11 & & 61.15 & & 37.82 & & 52.31 & & 18.67 & & 22.76 & \\
\hline Fs (\%) & 32.42 & & 39.41 & & 61.73 & & 26.90 & & 28.54 & & 29.01 & & 37.60 & & 47.79 & & 62.47 & \\
\hline State & & & & & & & & & Goi & & & & & & & & & \\
\hline Sample & & & & $\mathrm{TM}$ & 102 & & & & & & & & TM1 & & & & & \\
\hline & Mean & $\sigma$ & Mean & $\sigma$ & Mean & $\sigma$ & Mean & $\sigma$ & Mean & $\sigma$ & Mean & $\sigma$ & Mean & $\sigma$ & Mean & $\sigma$ & Mean & $\sigma$ \\
\hline & $\begin{array}{l}\text { Core } \\
\text { Aug }\end{array}$ & $\mathrm{n}=8$ & $\begin{array}{l}\text { Interm } \\
\text { Aug }\end{array}$ & $\mathrm{n}=10$ & $\begin{array}{l}\text { Rim } \\
\text { Aug }\end{array}$ & $\mathrm{n}=4$ & $\begin{array}{l}\text { Rim } \\
\text { Pgt }\end{array}$ & $\mathrm{n}=1$ & $\begin{array}{l}\text { Core } \\
\text { Aug }\end{array}$ & $\mathrm{n}=7$ & $\begin{array}{c}\text { Interm } \\
\text { Aug }\end{array}$ & $\mathrm{n}=8$ & $\begin{array}{l}\text { Rim } \\
\text { Aug }\end{array}$ & $\mathrm{n}=1$ & $\begin{array}{l}\text { Rim } \\
\text { Pgt }\end{array}$ & $\mathrm{n}=2$ & & \\
\hline $\mathrm{SiO}_{2}$ & 49.71 & 0.55 & 49.64 & 0.28 & 49.82 & 0.34 & 50.32 & & 49.98 & 0.15 & 49.78 & 0.13 & 48.35 & & 49.28 & 0.69 & & \\
\hline $\mathrm{Al}_{2} \mathrm{O}_{3}$ & 2.26 & 0.24 & 2.04 & 0.32 & 1.23 & 0.53 & 0.58 & & 1.42 & 0.02 & 1.22 & 0.04 & 0.42 & & 0.66 & 0.23 & & \\
\hline $\mathrm{FeO}$ & 10.34 & 0.47 & 11.32 & 0.86 & 16.09 & 3.42 & 23.46 & & 14.62 & 0.12 & 16.62 & 0.57 & 22.50 & & 26.95 & 4.65 & & \\
\hline $\mathrm{MnO}$ & 0.25 & 0.03 & 0.27 & 0.04 & 0.37 & 0.05 & 0.55 & & 0.39 & 0.02 & 0.43 & 0.01 & 0.55 & & 0.71 & 0.13 & & \\
\hline $\mathrm{MgO}$ & 15.81 & 0.40 & 15.34 & 0.36 & 12.30 & 2.70 & 20.17 & & 14.17 & 0.22 & 13.15 & 0.31 & 8.31 & & 14.09 & 2.52 & & \\
\hline $\mathrm{CaO}$ & 18.95 & 0.47 & 18.71 & 0.34 & 17.64 & 1.21 & 3.13 & & 17.51 & 0.23 & 16.93 & 0.25 & 18.11 & & 6.78 & 1.36 & & \\
\hline $\mathrm{Na}_{2} \mathrm{O}$ & 0.27 & 0.02 & 0.29 & 0.02 & 0.57 & 0.63 & 0.04 & & 0.24 & 0.02 & 0.21 & 0.01 & 0.24 & & 0.09 & 0.07 & & \\
\hline $\mathrm{TiO}_{2}$ & 1.10 & 0.12 & 1.13 & 0.09 & 0.67 & 0.34 & 0.44 & & 0.87 & 0.01 & 0.78 & 0.01 & 0.38 & & 0.44 & 0.09 & & \\
\hline $\mathrm{NiO}$ & 0.04 & 0.03 & 0.02 & 0.03 & 0.04 & 0.03 & 0.05 & & 0.01 & 0.01 & 0.02 & 0.01 & 0.00 & & 0.02 & 0.02 & & \\
\hline $\mathrm{Cr}_{2} \mathrm{O}_{3}$ & 0.14 & 0.07 & 0.05 & 0.06 & 0.01 & 0.01 & 0.02 & & 0.00 & 0.00 & 0.01 & 0.00 & 0.00 & & 0.00 & 0.00 & & \\
\hline $\mathrm{H}_{2} \mathrm{O}$ & 1.12 & & 1.18 & & 1.25 & & 1.25 & & 0.77 & & 0.84 & & 1.12 & & 0.97 & & & \\
\hline Total & 100.00 & & 100.00 & & 100.00 & & 100.00 & & 100.00 & & 100.00 & & 100.00 & & 100.00 & & & \\
\hline $\begin{array}{l}\text { Wo } \\
(\%)\end{array}$ & 38.50 & & 38.10 & & 37.05 & & 6.27 & & 35.77 & & 34.88 & & 37.99 & & 14.13 & & & \\
\hline En (\%) & 44.70 & & 43.47 & & 35.95 & & 56.20 & & 40.28 & & 37.70 & & 24.26 & & 40.86 & & & \\
\hline Fs $(\%)$ & 16.80 & & 18.43 & & 27.00 & & 37.54 & & 23.95 & & 27.43 & & 37.75 & & 45.01 & & & \\
\hline
\end{tabular}

$\sigma=$ standart deviation, Interm $=$ intermediary, $\mathrm{Aug}=$ augite and Pgt $=$ pigeonite and $\mathrm{n}=$ number of analyses 

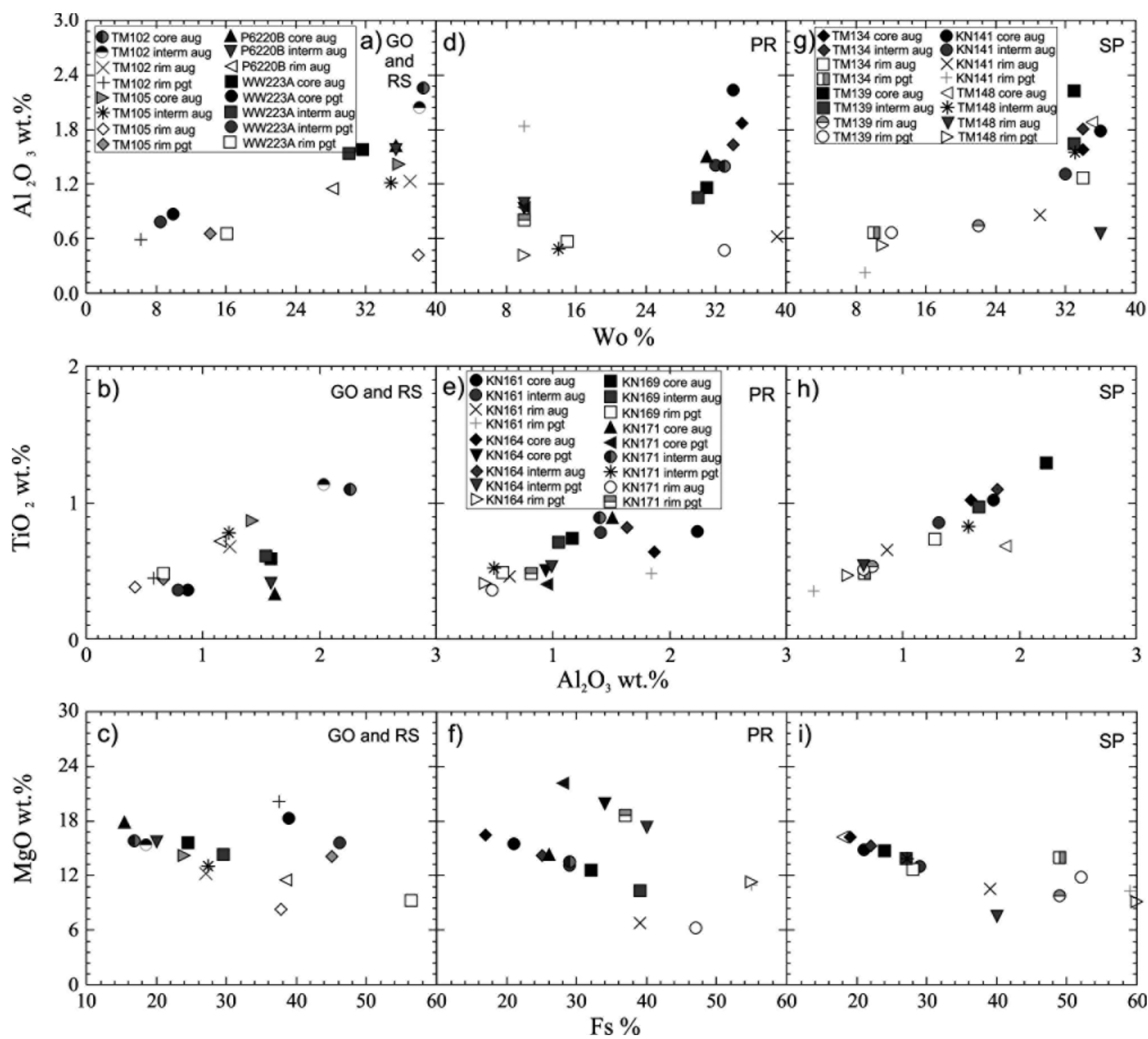

Figure $7-\mathrm{Al}_{2} \mathrm{O}_{3}$ wt.\% versus $\mathrm{Wo} \%, \mathrm{TiO}_{2}$ wt.\% versus $\mathrm{Al}_{2} \mathrm{O}_{3}$ wt.\% and $\mathrm{MgO}$ wt.\% versus $\mathrm{Fs} \%$ for clinopyroxene average microanalyses from EPMA in core, intermediate and rim zones from São Paulo (SP), Paraná (PR), Goiás (GO) and Rio Grande do Sul (RS). Aug = augite and pgt = pigeonite.

quantify the elements concentration of the melt and the analyses results of LA-ICP-MS quantified the values in ppm of the trace elements of core, intermediate and rim zones in those minerals. Table 4 shows data compilation of partition coefficients for elements in basaltic andesite and basalt which were studied by several authors in the $60 \mathrm{~s}$ and $90 \mathrm{~s}$. It is worth mentioning that the $\mathrm{K}_{\mathrm{D}}$ determination in those decades was carried out by separating minerals of interest from the rocks for further analyses by means of AAS and XRF according to available technology in that time.

The coefficient of partition in plagioclase and clinopyroxene crystals in this study are based on the use of advanced spot techniques of analyses, avoiding inclusions, zones and fractures which may interfere in the values, thus obtaining $\mathrm{K}_{\mathrm{D}}$ not only in the grain as a whole but in several portions of the crystal, allowing the understanding of the grain genesis and trace elements interaction in the temperature and crystallization ranges in these minerals.

Characteristic X-ray maps were done with the help of EPMA which resulted in different maps: K, Na, $\mathrm{Ca}, \mathrm{Fe}, \mathrm{Mg}$, Ti and $\mathrm{Al}$. In the present study, we selected one clinopyroxene crystal (KN141 - $\mathrm{Ca}$ and $\mathrm{Mg}$ elements) and one plagioclase crystal (KN161 - Ca and $\mathrm{Na}$ ) to illustrate these maps. The characteristic X-ray map and backscattering images showing the EPMA analyses and LA-ICP-MS for different trace elements are shown in figure 9 . 

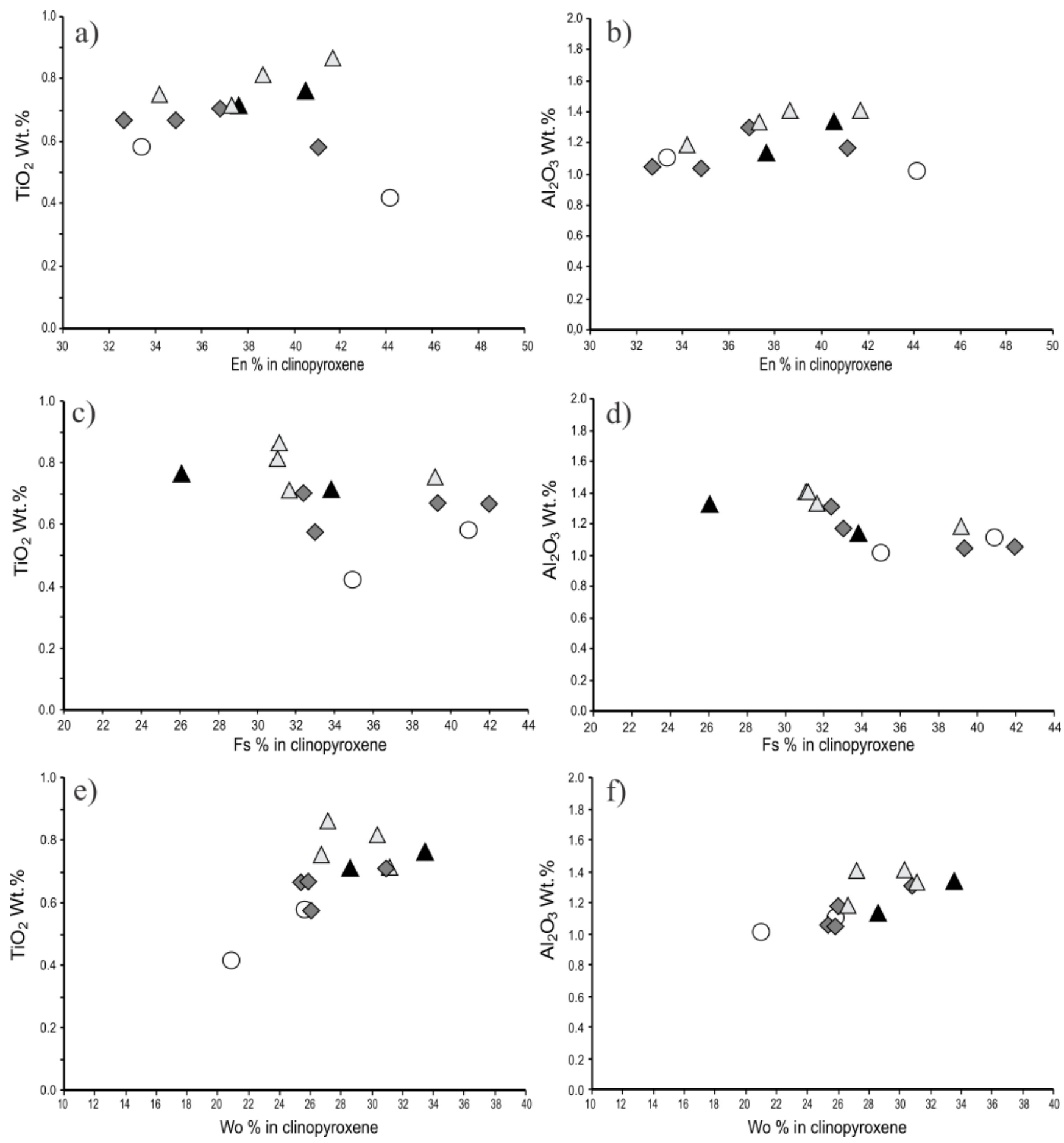

Figure 8 - Clinopyroxene average values of $\mathrm{TiO}_{2}$, and $\mathrm{Al}_{2} \mathrm{O}_{3}$ wt.\% versus En, Fs and Wo \% content in sills of Serra Geral Formation, Paraná Basin. Black triangle: clinopyroxene of Goiás; gray triangle: São Paulo; gray lozenge: Paraná; white circle: Rio Grande do Sul.

Plagioclase In the studied rocks, plagioclase displays zonation in most of the analyzed crystals, identifying core, intermediate and rim. Those zonations were previously identified by microscope and backscattering images.

The LA-ICP-MS microanalyses in core, intermediate and rim zones in plagioclase crystals of Serra Geral Formation sills, Paraná Basin, were able to identify variations in the partition coefficient shown in table 5. In this table, only the mean of two analyzed plagioclase crystals of KN161 sample from Paraná state and the mean of two augite crystals (KN141) from São Paulo state are discussed.

The most compatible element with plagioclase is $\mathrm{Sr}$, followed by $\mathrm{Eu}$ and $\mathrm{Pb}$ (LILE) with compatible values at rim in some of the plagioclase. 

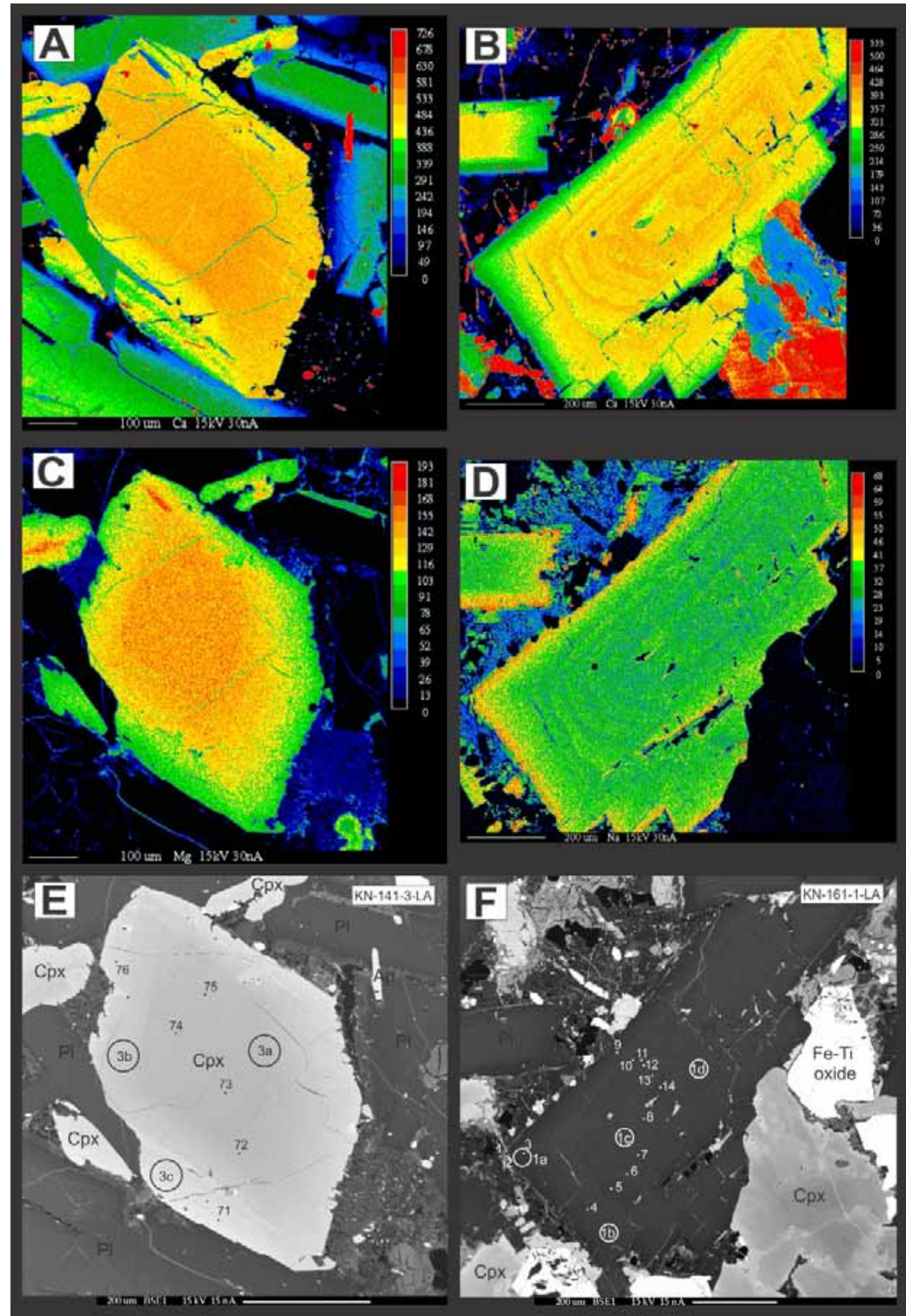

Figure 9 - Characteristic X-ray map composition of clinopyroxene $(A)=C a$ and $(C)=M g$ and plagioclase $(B)=$ $C a$ and $(D)=N a$ before EPMA and LA-ICP-MS microanalyses. Backscattered electron images of clinopyroxene (E) and plagioclase (F). Open circles show LA-ICP-MS analyses locations. The filled circles show EPMA analyses locations. Recommendations by the IUGS Subcommission on the Systematics of Metamorphic Rocks: Pl = plagioclase; $C p x=$ clinopyroxene; $O p=$ opaque minerals and Ap = apatite. 
Table 4 - Mineral/melt partition coefficients for basalt and basaltic andesite liquids compiled from 37 published experimental studies. Blank = not found.

\begin{tabular}{|c|c|c|c|c|c|c|c|c|c|}
\hline & \multicolumn{2}{|c|}{ Clinopyroxene } & \multicolumn{2}{|c|}{ Plagioclase } & & \multicolumn{2}{|c|}{ Clinopyroxene } & \multicolumn{2}{|c|}{ Plagioclase } \\
\hline & Basalt & $\begin{array}{l}\text { Basaltic } \\
\text { andesite }\end{array}$ & Basalt & $\begin{array}{l}\text { Basaltic } \\
\text { andesite }\end{array}$ & & Basalt & $\begin{array}{l}\text { Basaltic } \\
\text { andesite }\end{array}$ & Basalt & $\begin{array}{l}\text { Basaltic } \\
\text { andesite }\end{array}$ \\
\hline $\mathrm{Sc}$ & $1.42^{(1)}-3.3^{(2)}$ & $2.2-3.5^{(3)}$ & $0.008^{(2)}-0.1^{(4)}$ & & $\mathrm{Ce}$ & $\begin{array}{c}0.017^{(26)} \\
1.05^{(28)}\end{array}$ & $\begin{array}{c}0.09^{(29)}- \\
0.58^{(20)}\end{array}$ & $0.016^{(17)}-0.2^{(11)}$ & $0.06^{(8)}-0.14^{(7)}$ \\
\hline $\mathrm{Ti}$ & $0.37^{(1)}-0.45^{(5)}$ & $0.19-0.34^{(3)}$ & $0.038^{(6)}$ & $0.04-0.057^{(7)}$ & $\operatorname{Pr}$ & $0.04^{(30)}-0.626^{(9)}$ & $\begin{array}{c}0.635- \\
1.012^{(20)}\end{array}$ & $\begin{array}{c}0.063^{(19)}- \\
0.17^{(11)}\end{array}$ & $0.035-0.13^{(7)}$ \\
\hline V & $0.74^{(6)}-4.82^{(1)}$ & $0.5-7.2^{(8)}$ & $0.01^{(6)}$ & $0.022-0.032^{(7)}$ & $\mathrm{Nd}$ & $0.06^{(30)}-0.69^{(28)}$ & $\begin{array}{c}0.5^{(8)}- \\
1.31^{(20)}\end{array}$ & $\begin{array}{c}0.014^{(17)}- \\
0.168^{(19)}\end{array}$ & $\begin{array}{c}0.026^{(7)}- \\
0.15^{(8)}\end{array}$ \\
\hline $\mathrm{Cr}$ & $2.94-26.91^{(9)}$ & $9.7^{(3)}-70^{(8)}$ & $0.02^{(6)}-0.6^{(4)}$ & $0.075^{(7)}$ & $\mathrm{Sm}$ & $0.462^{(5)}$ & $\begin{array}{l}0.50^{(36)}- \\
0.445^{(37)}\end{array}$ & $\begin{array}{c}0.067^{(36)}{ }_{-} \\
0.072^{(37)}\end{array}$ & $0.0394^{(37)}$ \\
\hline $\mathrm{Mn}$ & $0.55-1.31^{(12)}$ & $1.2-1.8^{(3)}$ & $0.04^{(13)}-0.07^{(6)}$ & & $\mathrm{Eu}$ & $0.2^{(30)}-0.87^{(18)}$ & $\begin{array}{c}0.09^{(8)} \\
1.2^{(20)} \\
\end{array}$ & $\begin{array}{c}0.062^{(19)}- \\
1.526^{(18)}\end{array}$ & $0.06^{(8)}-0.79^{(7)}$ \\
\hline Co & $0.68-1.22^{(12)}$ & $1.2^{(10)}-2.4^{(3)}$ & $0.07-0.5^{(4)}$ & & Gd & $0.24-0.84^{(31)}$ & $\begin{array}{l}0.778- \\
1.24^{(20)}\end{array}$ & $\begin{array}{l}0.004^{(7)}- \\
0.066^{(11)}\end{array}$ & $\begin{array}{c}0.016^{(7)}- \\
0.08^{(8)}\end{array}$ \\
\hline $\mathrm{Ni}$ & $1.2-10^{(14)}$ & $4^{(10)}-10^{(3)}$ & $0.04-0.5^{(4)}$ & & $\mathrm{Tb}$ & $0.28-1.124^{(9)}$ & $\begin{array}{c}0.57^{(10)}- \\
1.33^{(20)}\end{array}$ & $0.013^{(2)}-0.11^{(4)}$ & $0.04-0.061^{(7)}$ \\
\hline $\mathrm{Cu}$ & $\begin{array}{c}0.071^{(2)} \_ \\
0.36^{(15)}\end{array}$ & $\begin{array}{c}0.05^{(10)} \\
0.69^{(3)} \\
\end{array}$ & $\begin{array}{c}0.004^{(2)}{ }_{-} \\
0.17^{(6)}\end{array}$ & $0.07-0.38^{(3)}$ & Dy & $\begin{array}{c}0.256^{(32)}- \\
1.18^{(28)}\end{array}$ & $\begin{array}{l}0.764- \\
1.09^{(20)}\end{array}$ & $\begin{array}{c}0.018^{(33)_{-}} \\
0.314^{(19)}\end{array}$ & $\begin{array}{c}0.011^{(7)}{ }_{-} \\
0.06^{(8)}\end{array}$ \\
\hline $\mathrm{Zn}$ & $0.5^{(6)}$ & $0.24-0.31^{(10)}$ & $0.11^{(6)}-0.18^{(13)}$ & & Ho & $0.3-1^{(30)}$ & $\begin{array}{l}0.712- \\
1.13^{(20)}\end{array}$ & $\begin{array}{l}0.011^{(2)}{ }_{-} \\
0.048^{(11)}\end{array}$ & \\
\hline $\mathrm{Rb}$ & $\begin{array}{c}0.011^{(11)}- \\
0.13^{(4)}\end{array}$ & $0.01-0.04^{(8)}$ & $0.016^{(16)}-0.3^{(4)}$ & $\begin{array}{c}0.008^{(7)}- \\
0.19^{(8)}\end{array}$ & $\mathrm{Er}$ & $\begin{array}{c}0.259^{(32)}- \\
1.17^{(28)}\end{array}$ & $\begin{array}{c}0.615- \\
0.922^{(20)}\end{array}$ & $\begin{array}{c}0.018^{(19)}- \\
0.041^{(11)}\end{array}$ & $\begin{array}{c}0.005^{(7)}- \\
0.06^{(27)}\end{array}$ \\
\hline $\mathrm{Sr}$ & $\begin{array}{c}0.04^{(17)}- \\
0.44^{(18)}\end{array}$ & $0.6-0.9^{(8)}$ & $1.55^{(17)}-10^{(4)}$ & $1.3^{(8)}-3.5^{(7)}$ & $\mathrm{Tm}$ & $0.2^{(30)}-1.047^{(9)}$ & & $0.036^{(11)}$ & \\
\hline Y & $0.29-1.71^{(9)}$ & $0.66-1.12^{(20)}$ & $0.023-0.03^{(19)}$ & $0.01-0.038^{(7)}$ & $\mathrm{Yb}$ & $\begin{array}{c}0.178^{(23)}- \\
1.14^{(31)}\end{array}$ & $\begin{array}{c}0.09- \\
1.03^{(20)}\end{array}$ & $\begin{array}{c}0.004^{(17)}- \\
0.04^{(34)}\end{array}$ & $0.004^{(7)}-0.3^{(8)}$ \\
\hline $\mathrm{Zr}$ & $0.001^{(9)}-0.7^{(21)}$ & $\begin{array}{c}0.235- \\
0.382^{(20)}\end{array}$ & $0.01^{(22)}-0.27^{(4)}$ & $0.0009^{(7)}$ & $\mathrm{Lu}$ & $0.006^{(9)}-1.12^{(28)}$ & $0.55-0.6^{(10)}$ & $\begin{array}{c}0.007^{(19)}- \\
0.037^{(33)}\end{array}$ & $\begin{array}{c}0.031- \\
0.043^{(27)}\end{array}$ \\
\hline $\mathrm{Nb}$ & $\begin{array}{c}0.004- \\
0.065^{(21)}\end{array}$ & $\begin{array}{c}0.025- \\
0.121^{(20)}\end{array}$ & $0.01^{(11)}$ & $0.008-0.045^{(7)}$ & $\mathrm{Hf}$ & $0.004^{(9)}-0.48^{(4)}$ & $\begin{array}{c}0.3^{(10)}- \\
0.589^{(20)}\end{array}$ & $0.01^{(11)}-0.13^{(4)}$ & $0.02^{(10)}$ \\
\hline Mo & & & & $0.39^{(7)}$ & $\mathrm{Ta}$ & $0.011-0.261^{(21)}$ & $0.013^{(36)}$ & $0.04-0.08^{(4)}$ & $0.027-0.11^{(7)}$ \\
\hline Cs & $\begin{array}{c}0.001^{(23)}- \\
0.13^{(4)}\end{array}$ & $\begin{array}{c}0.026- \\
0.048^{(20)}\end{array}$ & $0.13^{(4)}-1^{(13)}$ & $0.022-0.067^{(7)}$ & $\mathrm{Pb}$ & $\begin{array}{c}0.005^{(24)} \\
0.014^{(5)}\end{array}$ & $\begin{array}{c}0.102- \\
0.126^{(20)}\end{array}$ & $0.36^{(11)}-0.76^{(13)}$ & $0.18-1.07^{(7)}$ \\
\hline $\mathrm{Ba}$ & $\begin{array}{c}0.0001^{(24)}- \\
0.05^{(5)}\end{array}$ & $\begin{array}{c}0.002^{(25)}- \\
0.2^{(8)}\end{array}$ & $0.183^{(19)}-3.6^{(4)}$ & $0.03^{(8)}-1.45^{(7)}$ & Th & $\begin{array}{c}0.0003^{(11)}{ }_{-} \\
0.04^{(4)}\end{array}$ & $\begin{array}{c}0.019- \\
0.038^{(20)}\end{array}$ & $0.05-0.09^{(4)}$ & $0.01^{(10)} 0.19^{(7)}$ \\
\hline $\mathrm{La}$ & $\begin{array}{c}0.002^{(26)}{ }_{-} \\
0.21^{(9)}\end{array}$ & $\begin{array}{c}0.12^{(10)_{-}} \\
0.4^{(20)}\end{array}$ & $0.02^{(17)}-0.3^{(11)}$ & $\begin{array}{l}0.075^{(7)}- \\
0.227^{(27)}\end{array}$ & $\mathrm{U}$ & $\begin{array}{c}0.0003^{(23)}- \\
0.06^{(35)}\end{array}$ & $\begin{array}{c}0.02^{(20)_{-}} \\
0.04^{(10)}\end{array}$ & $0.06-0.1^{(4)}$ & $0.01^{(10)}-0.34^{(7)}$ \\
\hline
\end{tabular}

(1)=Jenner et al. 1993; (2)=Paster et al. 1974; ${ }^{(3)}=$ Ewart et al. 1973; ${ }^{(4)}=$ Villemant et al. 1981; (5)=Hauri et al. 1994; (6)=Bougault \& Hekinian, 1974; ${ }^{(7)}=$ Dunn \& Sen, 1994; ${ }^{(8)}=$ Reid, 1983; ${ }^{(9)}=$ Skulski et al. 1994; ${ }^{(10)}=$ Dostal et al. 1983; ${ }^{(1)}=$ McKenzie \& O'Nions, 1991; ${ }^{(12)}=$ Dale \& Henderson, 1972; ${ }^{(13)}=$ Kravuchuk et al. 1981; ${ }^{(14)}=$ Duke, 1976; ${ }^{(15)}=$ Hart \& Dunn, 1993; ${ }^{(16)}=$ Matsui et al. 1977; ${ }^{(17)}=$ McKay et al. 1994; ${ }^{(18)}=$ Sun et al. 1974; ${ }^{(19)}=$ Bindeman et al. 1998; ${ }^{(20)}=$ Larsen, 1979; ${ }^{(21)}=$ Forsythe et al. 1994; ${ }^{(22)}=$ McCallum \& Charette, 1978; ${ }^{(23)}=$ Watson et al. 1987; ${ }^{(24)}=$ Beattie, 1993; ${ }^{(25)}=$ Hart \& Brooks, 1974; ${ }^{(26)}=$ Frey, 1969; ${ }^{(27)}=$ Drake \& Weill, 1975; ${ }^{(28)}=$ Nagasawa, 1973; ${ }^{(29)}=$ Gaetani $\&$ Grove, 1995; ${ }^{(30)}=$ Irving \& Frey, 1984; ${ }^{(31)}=$ Hack et al. 1994; ${ }^{(32)}=$ Sobolev et al. 1996; ${ }^{(33)}=$ Schnetzler \& Philpotts, 1970; ${ }^{(34)}=$ Ringwood, 1970, ${ }^{(35)}=$ Benjamin et al. 1978, ${ }^{(36)}=$ Arth, 1976 and ${ }^{(37)}=$ Fujimaki et al. 1984.

Table 6 shows the plagioclase compatible elements in the different analyzed samples. The mean values for partition coefficients for plagioclase analyses are shown in table 7. Only two samples (KN164 and KN161) present negative anomalies of REE, different from the other samples which show low and positive REE concentrations.
The concentrations in variation in elements $\mathrm{Sr}$, $\mathrm{Ba}, \mathrm{Eu}$ and $\mathrm{Pb}$ from core to rim are common for all the plagioclases with a normal crystallization. This fact occurs because of the valence similarity and ionic radius between the $\mathrm{Ca}^{2+}(0.99 \AA$ in eightfold coordination) and $\mathrm{Na}^{+}(0.95 \AA$ in eightfold coordination) with this elements. Ca low concentrations at rim of plagioclase 
minerals (Fig. 9B) would correlate to low $\mathrm{K}_{\mathrm{D}}$ for $\mathrm{Sr}$ and $\mathrm{Eu}$, but in analyses of those rims we have found opposite, we found high Na concentrations (Fig. 9D) related to larger $\mathrm{K}_{\mathrm{D}}$ at rim.

Aiming to identify the zones to which the element $\mathrm{K}_{\mathrm{D}}$ is related, binary diagrams were used (Fig. 10) for showing the distribution in core, intermediate and rim for $\mathrm{Ca}$ and $\mathrm{Na}$. The partition coefficients of $\mathrm{Sr}, \mathrm{Eu}, \mathrm{Ba}$ and $\mathrm{Pb}$ versus $\mathrm{An} \%$ content in plagioclase crystals, and higher values of $A n$ are in the core of the crystal, the lowest values of An are related to the rim of the mineral. Figure 10a shows the $\mathrm{K}_{\mathrm{D}} \mathrm{Sr}$, consistent element in all samples with negative correlation in most of them, that is, the $\mathrm{K}_{\mathrm{D}} \mathrm{Sr}$ increase from core to rim, except from TM105 sample, this suggests that for Sr the substitution element is related mostly by replacement of $\mathrm{Na}$ instead of $\mathrm{Ca}$. The partition coefficient of Eu (Fig. 10b) shows that only half of the samples are compatible with this element in plagioclase, showing negative correlation with the An content, and the higher values of $\mathrm{K}_{\mathrm{D}} \mathrm{Eu}$ are related to the rim of this mineral, with affinity for $\mathrm{Na}$. This case resembles the diagram $K_{D}$ Ba versus An\%, with higher values for the rim. $\mathrm{Pb}$ is compatible in only five samples (core to rim), with negative to neutral correlations (Fig. 10d). Because the valence of $\mathrm{Pb}^{4+}$ ( $0.84 \AA$ in sixfold coordination) is very different from the physical and chemical characteristics of $\mathrm{Ca}$ and $\mathrm{Na}$, no satisfactory correlation of these elements was found in plagioclase, only with melt composition. Other variables, such as temperature, pressure, liquid composition, melt composition and $f \mathrm{O}_{2}$.can modify the partition coefficient in plagioclase and clinopyroxene.

Clinopyroxene Clinopyroxene has a larger number of elements compared with plagioclase in the same sample. This is because of the variety between the various stages of clinopyroxene and diverse elements of its chemical formula $\left(\mathrm{Mg}_{2} \mathrm{Si}_{2} \mathrm{O}_{6}-\mathrm{CaMgSi}_{2} \mathrm{O}_{6}-\right.$ $\left.\mathrm{CaFeSi}_{2} \mathrm{O}_{6}-\mathrm{Fe}_{2} \mathrm{Si}_{2} \mathrm{O}_{6}\right)$ in quadrilateral representation.
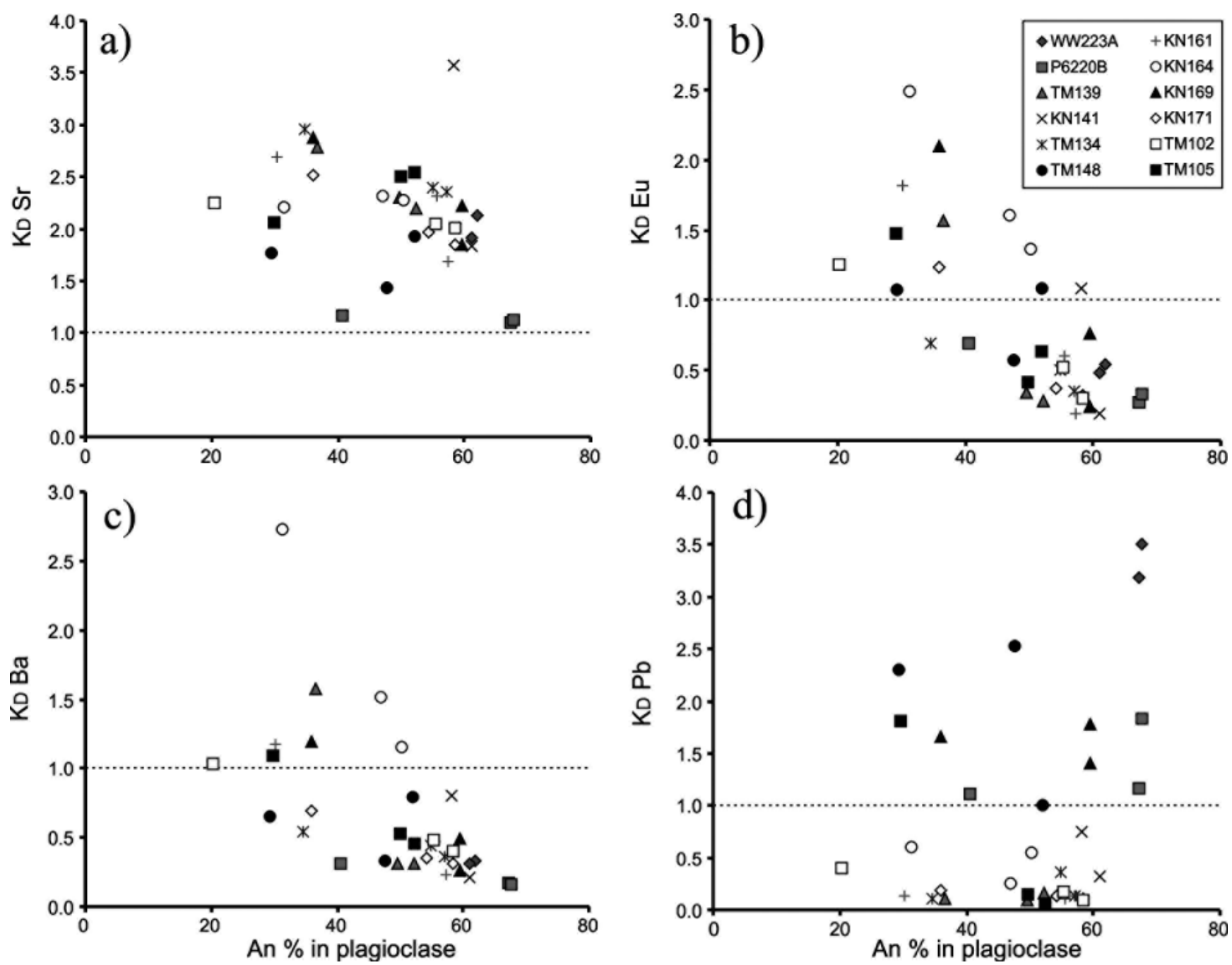

Figure 10 - Calculated partition coefficient (LA-ICP-MS) for a) Sr, b) Eu, c) Ba and d) Pb versus An\% plagioclase microanalyses (EPMA). The dashed line delineates the field among the compatible element zone and incompatible element zone. 


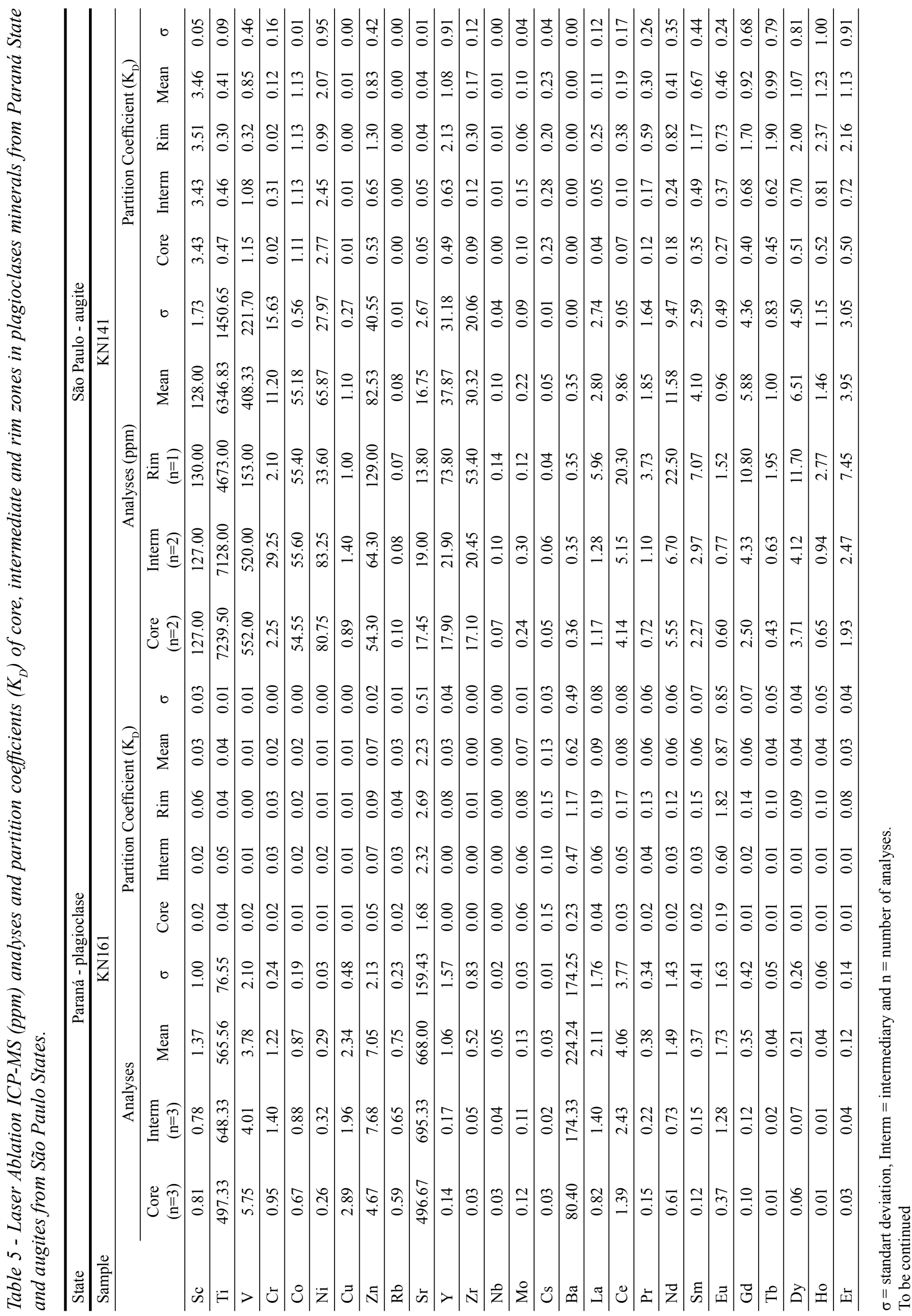




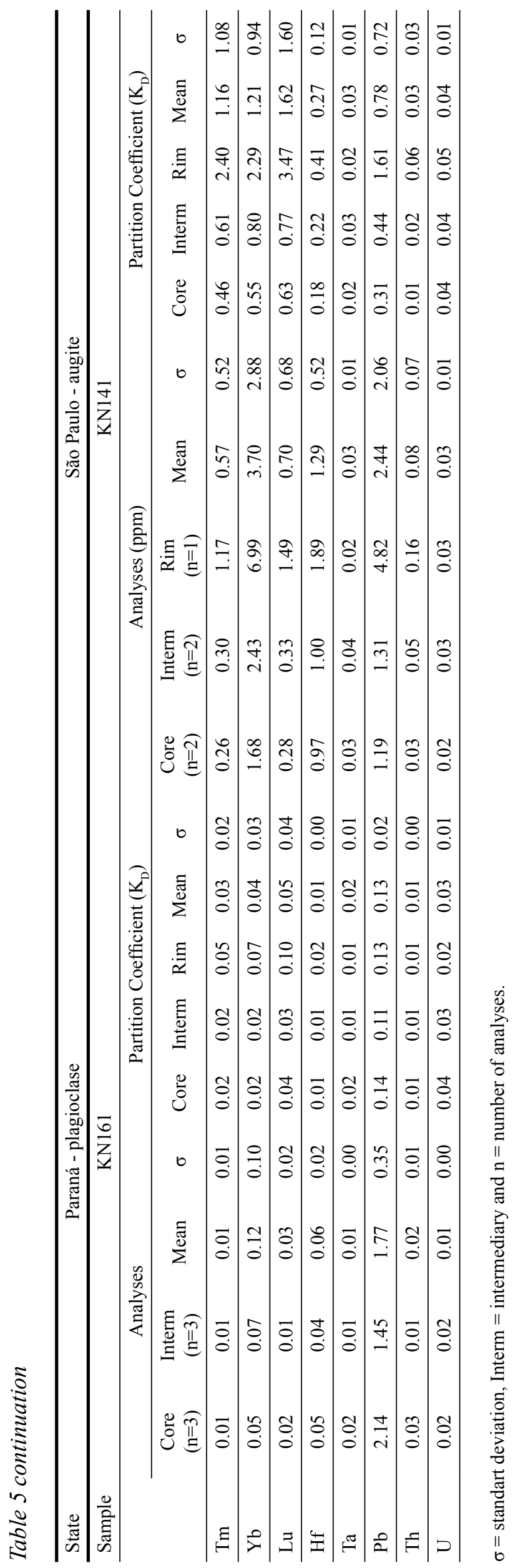

The substitution of trace elements is mainly for $\mathrm{Fe}^{2+}(0.64 \AA$ in sixfold coordination $), \mathrm{Mg}^{2+}(0.65 \AA$ in sixfold coordination), $\mathrm{Ca}^{2+}(0.99 \AA$ in eightfold coordination) and $\mathrm{Al}^{3+}(0.50 \AA$ in six or fourfold coordination). Different concentrations of $\mathrm{Ca}$ and $\mathrm{Mg}$ in the crystal of clinopyroxene are shown in figures 9A and $9 \mathrm{C}$ where the core of the clinopyroxene has the highest concentrations of $\mathrm{Mg}$ and $\mathrm{Ca}$ with reduction to the rim. Figure 9E shows the difference in brightness, dark to the core and lighter for the rim due to the larger concentrations of Fe. In the same figure, the LA-ICPMS and EPMA spots are also shown.

Table 8 shows the analyzed compatible elements in augite crystals.

A few crystals of clinopyroxene have rim of pigeonite and rare cases of core and intermediate zones, with values of $\mathrm{CaO}$ wt. $\%<7$. These areas of pigeonite were analyzed for trace elements, so it was possible to calculate the $\mathrm{K}_{\mathrm{D}}$ of these elements. However, the difficulty in analyzing the rim of pigeonite is due to the diameter of the laser ablation spot $(\sim 50 \mu \mathrm{m})$. Figure $2 \mathrm{~A}$ shows a very thin rim of pigeonite and figure $2 \mathrm{~B}$ shows a thicker rim.

Observing tables 9 and 10 it is possible to identify which clinopyroxene samples have higher concentrations of HREE than LREE. Table 10 shows the compatible elements values for these pigeonite zones. However, the higher values of $\mathrm{K}_{\mathrm{D}}$ of REE are found in the analyzed augite and smaller values of $\mathrm{K}_{\mathrm{D}}$ for the pigeonite.

Figure 11 shows $\mathrm{K}_{\mathrm{D}}$ values of $\mathrm{Sc}, \mathrm{Cr}, \mathrm{Ni}$, $\mathrm{Co}, \mathrm{V}, \mathrm{Zn}, \mathrm{Pb}$ and $\mathrm{Lu}$ versus $\mathrm{En} \%$ concentrations in clinopyroxene crystals. En\% shows positive correlation with $\mathrm{Ca}$ and $\mathrm{Al}$ and negative correlation with Fe. Thus, it was identified the probable replacement of these elements with the $\mathrm{Mg}, \mathrm{Ca}$ and Al concentrations according to the crystal core, intermediate and rim. The partition coefficients have positive correlations with En concentrations in clinopyroxene: $K_{D}$ Ni and $K_{D} V$ (Figs. 11c and 11e). The negative correlations are: $\mathrm{K}_{\mathrm{D}} \mathrm{Sc}, \mathrm{K}_{\mathrm{D}} \mathrm{Cr}, \mathrm{K}_{\mathrm{D}} \mathrm{Co}, \mathrm{K}_{\mathrm{D}}$ $\mathrm{Pb}$ and $\mathrm{K}_{\mathrm{D}} \mathrm{Lu}$ (Figs. 11a, 11b, 11d, 11g and 11h). The above values of $\mathrm{K}_{\mathrm{D}}>1$ for $\mathrm{Sc}$, Ni and Co show that these elements are compatible with the substitutions studied in clinopyroxene with $\mathrm{Mg}$ and $\mathrm{Fe}$. The values of $\mathrm{K}_{\mathrm{D}} \mathrm{Sc}\left(\mathrm{Sc}^{3+} 0.81 \AA\right.$ in sixfold coordination) suggest substitution for $\mathrm{Fe}$, related to an increase in $\mathrm{K}_{\mathrm{D}} \mathrm{Sc}$ to the rim mineral, except the TM139 and TM105 samples suggest that $\mathrm{Mg}$ substitution identified by the increase in $\mathrm{K}_{\mathrm{D}} \mathrm{Sc}$ values in the core (Fig. 11a). Distributions of partition coefficients are similar to figure $11 \mathrm{~d}$ when compared to figure 11a. Figure 11e $\left(\mathrm{K}_{\mathrm{D}} \mathrm{V}\right)$ has positive correlations with En\% (except P6220B, TM102 and TM134 samples negative correlations). Given the physical and chemical properties of $\mathrm{V}^{5+}(0.52 \AA$ in sixfold coordination), this element has a higher probability of substitution for $\mathrm{Al}^{3+}(0.50 \AA$ in sixfold coordination) than $\mathrm{Fe}$ and $\mathrm{Mg}$. The diagram in figure 11f shows increase in the $\mathrm{K}_{\mathrm{D}} \mathrm{Zn}^{2+}(0.74 \AA$ in sixfold coordination) from the core to the rim, due to probable 

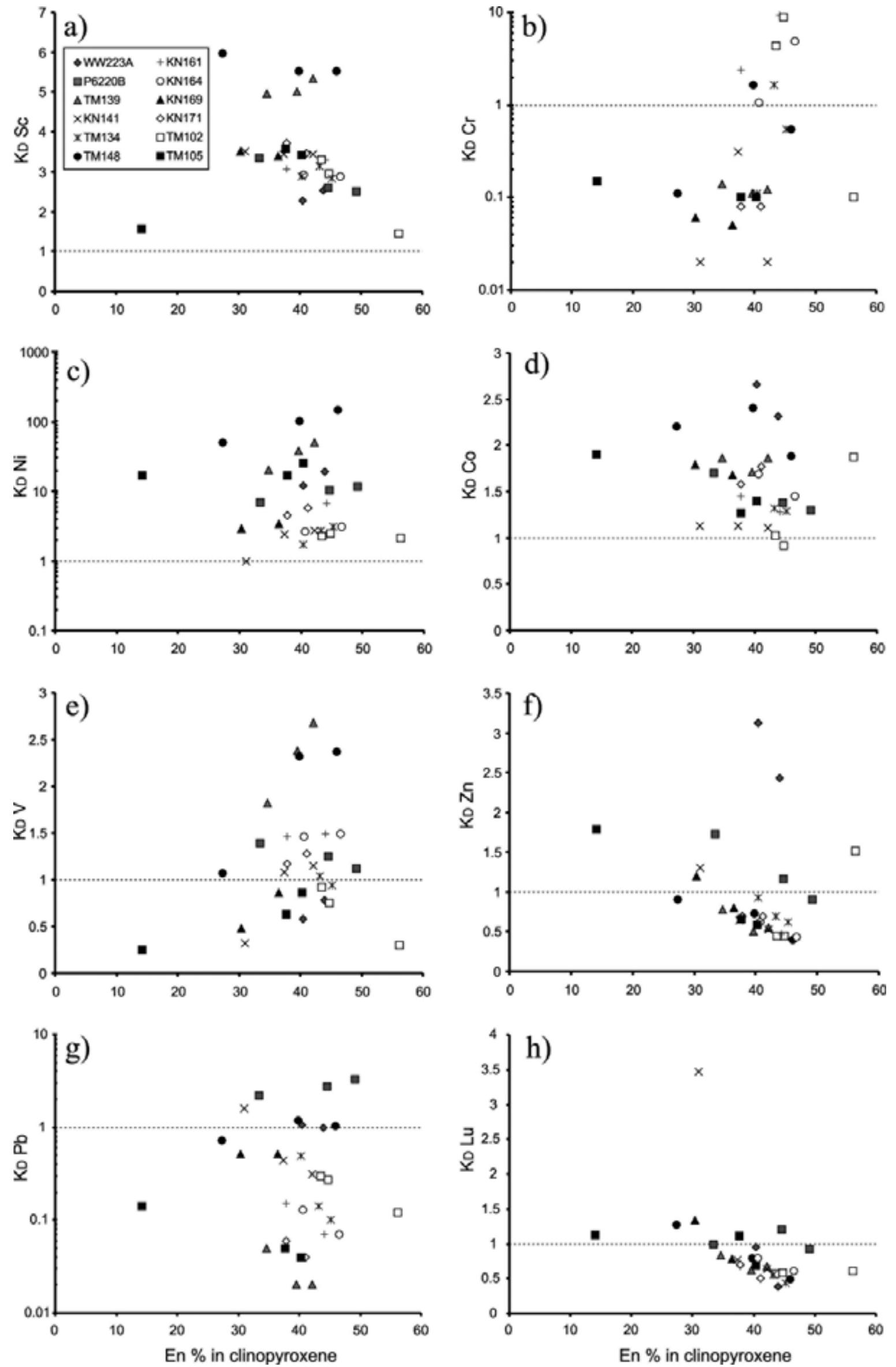

Figure 11 - Calculated partition coefficient (LA-ICP-MS) for a) Sc, b) Cr, c) Ni, d) Co, e) $\mathrm{V}, \mathrm{f}) \mathrm{Zn}, \mathrm{g}) \mathrm{Pb}$ and $h$ ) Lu versus En\% clinopyroxene microanalyses (EPMA). The dashed line delineates the field among the compatible element zone and incompatible element zone. 
Table 6 - Compatible elements zone in plagioclase crystals. Blank = not analyzed.

\begin{tabular}{|c|c|c|c|c|c|c|c|c|c|c|c|c|}
\hline \multirow{2}{*}{$\begin{array}{l}\text { Element } \\
\text { Zone }\end{array}$} & \multicolumn{3}{|c|}{$\mathrm{Sr}$} & \multicolumn{3}{|c|}{$\mathrm{Eu}$} & \multicolumn{3}{|c|}{$\mathrm{Ba}$} & \multicolumn{3}{|c|}{$\mathrm{Pb}$} \\
\hline & Core & Interm & Rim & Core & Interm & Rim & Core & Interm & Rim & Core & Interm & Rim \\
\hline WW223A & 2.13 & 1.91 & & \multicolumn{2}{|l|}{$<0.5$} & & \multicolumn{2}{|c|}{$<0.3$} & & 3.18 & 3.51 & \\
\hline P6220B & 1.1 & 1.13 & 1.16 & & $<0.7$ & & & $<0.3$ & & 1.16 & 1.83 & 1.11 \\
\hline KN141 & 1.83 & 3.57 & & 0.19 & 1.08 & & \multicolumn{2}{|c|}{$<0.8$} & & \multicolumn{2}{|c|}{$<0.7$} & \\
\hline TM134 & 2.57 & 0.33 & 2.95 & & $<0.7$ & & & $<0.5$ & & \multicolumn{3}{|c|}{$<0.3$} \\
\hline TM139 & 2.2 & 2.3 & 2.78 & 0.28 & 0.34 & 1.57 & 0.31 & 0.31 & 1.58 & \multicolumn{3}{|c|}{$<0.1$} \\
\hline TM148 & 1.92 & 1.43 & 1.77 & 1.08 & 0.57 & 1.07 & & $<0.8$ & & 1 & 2.53 & 2.3 \\
\hline KN161 & 1.68 & 2.32 & 2.69 & 0.19 & 0.60 & 1.82 & 0.22 & 0.46 & 1.17 & \multicolumn{3}{|c|}{$<1.3$} \\
\hline KN164* & 2.31 & 2.27 & 2.21 & 1.61 & 1.36 & 2.49 & 1.52 & 1.15 & 2.73 & \multicolumn{3}{|c|}{$<0.6$} \\
\hline KN169 & 1.84 & 2.22 & 2.88 & 0.24 & 0.76 & 2.1 & 0.26 & 0.49 & 1.19 & 1.78 & 1.4 & 1.66 \\
\hline KN171 & 1.84 & 1.97 & 2.51 & 0.32 & 0.37 & 1.23 & & $<0.9$ & & \multicolumn{3}{|c|}{$<0.2$} \\
\hline TM102 & 2.01 & 2.05 & 2.25 & 0.3 & 0.52 & 1.25 & 0.4 & 0.48 & 1.03 & \multicolumn{3}{|c|}{$<0.4$} \\
\hline TM105 & 2.5 & 2.54 & 2.06 & 0.42 & 0.64 & 1.48 & 0.54 & 0.47 & 1.11 & 0.16 & 0.1 & 1.8 \\
\hline
\end{tabular}

${ }^{*} \mathrm{KN} 164(\mathrm{La}$ core $=0.24, \mathrm{La}$ interm $=0.55, \mathrm{La} \mathrm{rim}=1.76 ; \mathrm{Ce}$ core $=0.18$, Ce interm $=0.48$, Ce rim $=1.62 ; \operatorname{Pr}$ core $=0.14, \operatorname{Pr}$ interm=0.42, Pr rim $=1.44 ; \mathrm{Nd}$ core $=0.1, \mathrm{Nd}$ interm $=0.37, \mathrm{Nd}$ rim $=1.53 ; \mathrm{Sm}$ core $=0.05, \mathrm{Sm}$ interm $=0.28, \mathrm{Sm}$ rim $=1.02$ ).

substitution for the Fe (except TM102 sample with significant increase in rim areas and intermediate for the core, substitution with $\mathrm{Mg}$ ). The diagram $\mathrm{K}_{\mathrm{D}} \mathrm{Lu}^{3+}$ (0.85 in sixfold coordination) versus En\% represents a similarity with the other elements HREE, suggesting negative correlations with the substitution for Fe.

CONCLUSIONS Based on geochemical relationships and in situ partition coefficients using Laser-ablation ICP-MS of plagioclase and clinopyroxene from sills in Serra Geral Formation, Paraná Basin, Brazil, we have identified change in composition of major and trace elements in core, intermediate and rim zones of these minerals.

Plagioclase is generally regarded to control partition coefficient of elements such as $\mathrm{Sr}$, Eu and $\mathrm{Pb}$. The crystallization of this mineral reflects the variations of major elements such as $\mathrm{K}_{2} \mathrm{O}, \mathrm{Al}_{2} \mathrm{O}_{3}, \mathrm{CaO}$ and $\mathrm{Na}_{2} \mathrm{O}$ wt.\% showing typical concentrations of basic and intermediate tholeiitic magmas.

According to mineral cooling the $\mathrm{Ca}^{2+} \mathrm{Al}^{3+} \leftrightarrow$ $\mathrm{Na}^{+} \mathrm{Si}^{4+}$ cation change by means of slow diffusion aiming at a rebalancing in $\mathrm{NaAlSi}_{3} \mathrm{O}_{8}-\mathrm{CaAl}_{2} \mathrm{Si}_{2} \mathrm{O}_{8}$ system, starting the crystallization in liquidus until its entire crystallization in the solidus line in binary system (1 atm) from Bowen (1928). The more Ca terms (anorthite, bytownite and labradorite) are formed in higher temperatures while the more $\mathrm{Na}$ (albite, oligoclase and andesine) in lower temperatures. The differences in composition of $\mathrm{Ca}$ and $\mathrm{Na}$ are followed by the partition coefficient values of several trace elements and REE.

Plagioclase has positive correlations for $\mathrm{Na}_{2} \mathrm{O}$ to the $\mathrm{K}_{\mathrm{D}} \mathrm{Sr}$ and $\mathrm{Eu}$ with higher values at the rim, suggesting a greater proportion of substitution of these elements for $\mathrm{Na}$. An explanation for that fact would be high $\mathrm{Na}$ and $\mathrm{K}$ concentrations at plagioclase minerals rim. Explanation could be that the magma oxidation state can affects the partition coefficient of $\mathrm{Eu}$, low $\mathrm{fO}_{2}$ increasing, because the ratio $\mathrm{Eu}^{2+} / \mathrm{Eu}^{3+}$ increasing with fusion, and the $\mathrm{Eu}^{2+}$ is much more compatible than $\mathrm{Eu}^{3+}$ in plagioclase basaltic (Best 2003).

The compositional variations of major elements such as $\mathrm{Fe}^{2+}, \mathrm{Mg}^{2+}, \mathrm{Ca}^{2+}$ and $\mathrm{Al}^{3+}$ in clinopyroxene influence the $\mathrm{K}_{\mathrm{D}}$ of elements such as $\mathrm{Sc}, \mathrm{V}, \mathrm{Cr}, \mathrm{Co}, \mathrm{Ni}$, $\mathrm{Zn}, \mathrm{Y}, \mathrm{HREE}$ and $\mathrm{Pb}$ in eightfold, sixfold and fourfold coordination.

The separation of the sills into high-Ti $\left(\mathrm{TiO}_{2}\right.$ $\geq 2 \%)$ and low- $\mathrm{Ti}\left(\mathrm{TiO}_{2} \leq 2 \%\right)$ is not only due to the abundance of Ti-magnetites and ilmenites, but also by lower concentrations of this element in plagioclase, as shown in this work.

The major elements and trace elements in core, intermediate and rim zones when combined with detailed chemical mapping provides new insights into how plagioclase and clinopyroxene grew in basic and intermediate sills in Paraná Basin, Brazil.

Additional studies in plagioclase and clinopyroxene are required to evaluate their genesis. We suggest the analyses of variables, such as $T, P, f \mathrm{O}_{2}$. 
Table 7 - Average partition coefficients from plagioclase sills in Serra Geral Formation, Paraná Basin, Brazil. Blank $=$ not analyzed .

Plagioclase

\begin{tabular}{|c|c|c|c|c|c|c|c|c|c|c|c|c|}
\hline \multirow{2}{*}{$\begin{array}{l}\text { State } \\
\text { Sample }\end{array}$} & \multicolumn{2}{|c|}{ Rio Grande do Sul } & \multicolumn{4}{|c|}{ São Paulo } & \multicolumn{4}{|c|}{ Paraná } & \multicolumn{2}{|c|}{ Goiás } \\
\hline & WW223A & P6220B & TM139 & KN141 & TM134 & TM148 & KN161 & KN164 & KN169 & KN171 & TM102 & TM105 \\
\hline Type & \multicolumn{3}{|c|}{ Basaltic andesite } & & & & & Basalt & & & & \\
\hline $\mathrm{Sc}$ & 0.02 & 0.02 & 0.07 & 0.03 & 0.01 & 0.03 & 0.03 & 0.02 & 0.02 & 0.02 & 0.02 & 0.02 \\
\hline $\mathrm{Ti}$ & 0.04 & 0.04 & 0.04 & 0.05 & 0.02 & 0.03 & 0.04 & 0.05 & 0.04 & 0.04 & 0.04 & 0.03 \\
\hline $\mathrm{V}$ & 0.01 & 0.02 & 0.01 & 0.01 & 0.01 & 0.01 & 0.01 & 0.02 & 0.01 & 0.01 & 0.01 & 0.01 \\
\hline $\mathrm{Cr}$ & & & 0.06 & 0.02 & 0.04 & 0.06 & 0.02 & 0.01 & 0.03 & 0.03 & 0.01 & 0.05 \\
\hline Co & 0.04 & 0.05 & 0.04 & 0.02 & 0.02 & 0.02 & 0.02 & 0.04 & 0.02 & 0.02 & 0.01 & 0.04 \\
\hline $\mathrm{Ni}$ & 0.06 & 0.09 & 0.28 & 0.01 & 0.02 & 0.37 & 0.01 & 0.02 & 0.01 & 0.01 & 0.01 & 0.40 \\
\hline $\mathrm{Cu}$ & 0.07 & 0.01 & 0.01 & 0.01 & 0.04 & 0.12 & 0.01 & 0.02 & 0.01 & 0.01 & 0.02 & 0.01 \\
\hline $\mathrm{Zn}$ & 0.18 & 0.19 & 0.11 & 0.07 & 0.06 & 0.09 & 0.07 & 0.09 & 0.07 & 0.07 & 0.07 & 0.10 \\
\hline $\mathrm{Rb}$ & 0.15 & 0.01 & 0.06 & 0.02 & 0.04 & 0.02 & 0.03 & 0.42 & 0.02 & 0.02 & 0.03 & 0.05 \\
\hline $\mathrm{Sr}$ & 2.02 & 1.13 & 2.43 & 2.70 & 1.95 & 1.71 & 2.23 & 2.26 & 2.32 & 2.10 & 2.11 & 2.37 \\
\hline $\mathrm{Y}$ & 0.02 & 0.01 & 0.07 & 0.01 & 0.01 & 0.00 & 0.03 & 0.21 & 0.01 & 0.00 & 0.01 & 0.01 \\
\hline $\mathrm{Zr}$ & 0.03 & 0.00 & 0.02 & 0.00 & 0.01 & 0.00 & 0.00 & 0.07 & 0.00 & 0.00 & 0.00 & 0.00 \\
\hline $\mathrm{Nb}$ & 0.05 & 0.00 & 0.01 & 0.00 & 0.01 & 0.00 & 0.00 & 0.03 & 0.00 & 0.00 & 0.00 & 0.00 \\
\hline Mo & 0.07 & 0.15 & 0.06 & 0.07 & 0.04 & 0.06 & 0.07 & 0.09 & 0.05 & 0.06 & 0.05 & 0.06 \\
\hline Cs & 0.15 & 0.02 & 0.03 & 0.14 & 0.05 & 0.04 & 0.13 & 0.27 & 0.03 & 0.07 & 0.12 & 0.09 \\
\hline $\mathrm{Ba}$ & 0.32 & 0.21 & 0.73 & 0.51 & 0.36 & 0.59 & 0.62 & 1.80 & 0.65 & 0.45 & 0.64 & 0.71 \\
\hline $\mathrm{La}$ & 0.11 & 0.06 & 0.10 & 0.08 & 0.06 & 0.07 & 0.09 & 0.85 & 0.07 & 0.05 & 0.10 & 0.07 \\
\hline $\mathrm{Ce}$ & 0.11 & 0.05 & 0.09 & 0.06 & 0.05 & 0.05 & 0.08 & 0.76 & 0.06 & 0.04 & 0.08 & 0.06 \\
\hline $\operatorname{Pr}$ & 0.08 & 0.03 & 0.08 & 0.05 & 0.04 & 0.03 & 0.06 & 0.67 & 0.04 & 0.03 & 0.06 & 0.04 \\
\hline $\mathrm{Nd}$ & 0.06 & 0.03 & 0.07 & 0.04 & 0.03 & 0.03 & 0.06 & 0.67 & 0.03 & 0.03 & 0.05 & 0.04 \\
\hline $\mathrm{Sm}$ & 0.05 & 0.02 & 0.07 & 0.03 & 0.02 & 0.02 & 0.06 & 0.45 & 0.02 & 0.02 & 0.03 & 0.02 \\
\hline $\mathrm{Eu}$ & 0.51 & 0.43 & 0.73 & 0.64 & 0.46 & 0.90 & 0.87 & 1.82 & 1.03 & 0.64 & 0.69 & 0.85 \\
\hline $\mathrm{Gd}$ & 0.03 & 0.03 & 0.07 & 0.03 & 0.02 & 0.01 & 0.06 & 0.36 & 0.01 & 0.01 & 0.02 & 0.01 \\
\hline $\mathrm{Tb}$ & 0.02 & 0.02 & 0.06 & 0.02 & 0.01 & 0.01 & 0.04 & 0.27 & 0.01 & 0.01 & 0.01 & 0.01 \\
\hline Dy & 0.02 & 0.01 & 0.07 & 0.01 & 0.01 & 0.01 & 0.04 & 0.28 & 0.01 & 0.01 & 0.01 & 0.01 \\
\hline Ho & 0.02 & 0.02 & 0.07 & 0.02 & 0.01 & 0.01 & 0.04 & 0.24 & 0.01 & 0.01 & 0.02 & 0.01 \\
\hline $\mathrm{Er}$ & 0.02 & 0.01 & 0.07 & 0.02 & 0.01 & 0.01 & 0.03 & 0.20 & 0.01 & 0.01 & 0.02 & 0.01 \\
\hline $\mathrm{Tm}$ & 0.03 & 0.04 & 0.08 & 0.04 & 0.01 & 0.02 & 0.03 & 0.16 & 0.02 & 0.01 & 0.04 & 0.02 \\
\hline $\mathrm{Yb}$ & 0.02 & 0.02 & 0.07 & 0.04 & 0.02 & 0.01 & 0.04 & 0.13 & 0.01 & 0.01 & 0.03 & 0.02 \\
\hline $\mathrm{Lu}$ & 0.03 & 0.04 & 0.09 & 0.03 & 0.02 & 0.02 & 0.05 & 0.13 & 0.02 & 0.02 & 0.04 & 0.02 \\
\hline $\mathrm{Hf}$ & 0.02 & 0.01 & 0.02 & 0.01 & 0.01 & 0.01 & 0.01 & 0.08 & 0.01 & 0.01 & 0.01 & 0.01 \\
\hline $\mathrm{Ta}$ & 0.05 & 0.03 & 0.10 & 0.02 & 0.01 & 0.01 & 0.02 & 0.07 & 0.01 & 0.01 & 0.02 & 0.01 \\
\hline $\mathrm{Pb}$ & 3.34 & 1.36 & 0.12 & 0.53 & 0.15 & 1.94 & 0.13 & 0.47 & 1.61 & 0.14 & 0.23 & 0.69 \\
\hline Th & 0.03 & 0.00 & 0.02 & 0.01 & 0.02 & 0.00 & 0.01 & 0.14 & 0.01 & 0.01 & 0.01 & 0.00 \\
\hline $\mathrm{U}$ & 0.13 & 0.01 & 0.02 & 0.04 & 0.03 & 0.02 & 0.03 & 0.14 & 0.03 & 0.02 & 0.04 & 0.02 \\
\hline
\end{tabular}


Table 8 - Average partition coefficients from clinopyroxenes sills in Serra Geral Formation, Paraná Basin, Brazil. Blank = not analyzed.

\begin{tabular}{|c|c|c|c|c|c|c|c|c|c|c|c|c|}
\hline \multicolumn{13}{|c|}{ Pyroxene } \\
\hline \multirow{2}{*}{$\begin{array}{l}\text { State } \\
\text { Sample }\end{array}$} & \multicolumn{2}{|c|}{ Rio Grande do Sul } & \multicolumn{4}{|c|}{ São Paulo } & \multicolumn{4}{|c|}{ Paraná } & \multicolumn{2}{|c|}{ Goiás } \\
\hline & WW223A & P6220B & TM139 & KN141 & TM134 & TM148 & KN161 & KN164 & KN169 & KN171 & TM102 & TM105 \\
\hline Type & \multicolumn{3}{|c|}{ Basaltic andesite } & \multicolumn{9}{|c|}{ Basalt } \\
\hline $\mathrm{Sc}$ & 2.52 & 2.82 & 4.97 & 3.46 & 2.95 & 5.67 & 3.18 & 2.89 & 3.45 & 3.60 & 3.17 & 3.50 \\
\hline $\mathrm{Ti}$ & 0.26 & 0.39 & 0.50 & 0.41 & 0.29 & 0.28 & 0.41 & 0.49 & 0.39 & 0.41 & 0.43 & 0.24 \\
\hline $\mathrm{V}$ & 0.78 & 1.26 & 2.29 & 0.85 & 0.95 & 1.92 & 1.45 & 1.47 & 0.67 & 1.23 & 0.84 & 0.75 \\
\hline $\mathrm{Cr}$ & & & 0.12 & 0.12 & 0.76 & 8.56 & 5.81 & 3.00 & 0.05 & 0.08 & 4.51 & 0.10 \\
\hline Co & 2.32 & 1.46 & 1.81 & 1.13 & 1.34 & 2.17 & 1.37 & 1.57 & 1.73 & 1.67 & 0.97 & 1.33 \\
\hline $\mathrm{Ni}$ & 19.18 & 9.67 & 35.73 & 2.07 & 2.51 & 98.45 & 5.67 & 2.86 & 3.13 & 5.10 & 2.19 & 20.83 \\
\hline $\mathrm{Cu}$ & 0.04 & 0.10 & 0.01 & 0.01 & 0.01 & 0.06 & 0.01 & 0.00 & 0.00 & 0.01 & 0.01 & 0.00 \\
\hline $\mathrm{Zn}$ & 2.43 & 1.26 & 0.61 & 0.83 & 0.75 & 0.68 & 0.59 & 0.53 & 1.00 & 0.69 & 0.75 & 0.62 \\
\hline $\mathrm{Rb}$ & 0.01 & 0.02 & 0.00 & 0.00 & 0.00 & 0.00 & 0.02 & 0.01 & 0.00 & 0.00 & 0.17 & 0.00 \\
\hline $\mathrm{Sr}$ & 0.03 & 0.07 & 0.05 & 0.04 & 0.05 & 0.03 & 0.04 & 0.06 & 0.04 & 0.05 & 0.07 & 0.06 \\
\hline $\mathrm{Y}$ & 0.35 & 0.77 & 0.57 & 1.08 & 0.51 & 0.54 & 0.53 & 0.58 & 0.87 & 0.56 & 0.60 & 0.73 \\
\hline $\mathrm{Zr}$ & 0.04 & 0.15 & 0.12 & 0.17 & 0.09 & 0.07 & 0.11 & 0.11 & 0.13 & 0.09 & 0.13 & 0.11 \\
\hline $\mathrm{Nb}$ & 0.01 & 0.10 & 0.00 & 0.01 & 0.00 & 0.00 & 0.02 & 0.03 & 0.00 & 0.00 & 0.00 & 0.00 \\
\hline Mo & 0.24 & 0.26 & 0.10 & 0.10 & 0.09 & 0.15 & 0.11 & 0.09 & 0.12 & 0.13 & 0.09 & 0.09 \\
\hline Cs & 0.03 & 0.09 & 0.06 & 0.23 & 0.10 & 0.08 & 0.22 & 0.11 & 0.06 & 0.16 & 0.17 & 0.09 \\
\hline $\mathrm{Ba}$ & 0.00 & 0.02 & 0.00 & 0.00 & 0.00 & 0.00 & 0.01 & 0.02 & 0.00 & 0.00 & 0.11 & 0.00 \\
\hline $\mathrm{La}$ & 0.02 & 0.09 & 0.04 & 0.11 & 0.04 & 0.03 & 0.08 & 0.06 & 0.06 & 0.04 & 0.09 & 0.06 \\
\hline $\mathrm{Ce}$ & 0.04 & 0.15 & 0.08 & 0.19 & 0.08 & 0.05 & 0.11 & 0.10 & 0.10 & 0.07 & 0.14 & 0.11 \\
\hline $\operatorname{Pr}$ & 0.07 & 0.19 & 0.13 & 0.30 & 0.13 & 0.08 & 0.16 & 0.17 & 0.16 & 0.13 & 0.19 & 0.19 \\
\hline $\mathrm{Nd}$ & 0.08 & 0.24 & 0.19 & 0.41 & 0.19 & 0.11 & 0.22 & 0.21 & 0.28 & 0.19 & 0.26 & 0.28 \\
\hline $\mathrm{Sm}$ & 0.11 & 0.44 & 0.35 & 0.67 & 0.32 & 0.26 & 0.35 & 0.40 & 0.47 & 0.31 & 0.43 & 0.45 \\
\hline $\mathrm{Eu}$ & 0.11 & 0.32 & 0.31 & 0.46 & 0.31 & 0.21 & 0.31 & 0.30 & 0.31 & 0.28 & 0.41 & 0.35 \\
\hline $\mathrm{Gd}$ & 0.28 & 0.60 & 0.48 & 0.92 & 0.43 & 0.36 & 0.51 & 0.51 & 0.63 & 0.45 & 0.50 & 0.60 \\
\hline $\mathrm{Tb}$ & 0.20 & 0.59 & 0.49 & 0.99 & 0.43 & 0.41 & 0.53 & 0.49 & 0.64 & 0.45 & 0.50 & 0.61 \\
\hline Dy & 0.36 & 0.71 & 0.60 & 1.07 & 0.54 & 0.52 & 0.61 & 0.58 & 0.84 & 0.60 & 0.61 & 0.77 \\
\hline Но & 0.34 & 0.83 & 0.67 & 1.23 & 0.58 & 0.68 & 0.63 & 0.68 & 0.90 & 0.62 & 0.67 & 0.81 \\
\hline $\mathrm{Er}$ & 0.38 & 0.89 & 0.61 & 1.13 & 0.53 & 0.69 & 0.62 & 0.59 & 0.84 & 0.57 & 0.55 & 0.74 \\
\hline $\mathrm{Tm}$ & 0.28 & 0.91 & 0.57 & 1.16 & 0.51 & 0.68 & 0.65 & 0.56 & 0.88 & 0.57 & 0.60 & 0.74 \\
\hline $\mathrm{Yb}$ & 0.48 & 1.05 & 0.62 & 1.21 & 0.58 & 0.66 & 0.62 & 0.63 & 0.93 & 0.60 & 0.61 & 0.79 \\
\hline $\mathrm{Lu}$ & 0.38 & 1.04 & 0.71 & 1.62 & 0.57 & 0.84 & 0.62 & 0.70 & 1.06 & 0.60 & 0.64 & 0.90 \\
\hline $\mathrm{Hf}$ & 0.12 & 0.19 & 0.21 & 0.27 & 0.18 & 0.13 & 0.21 & 0.18 & 0.21 & 0.16 & 0.20 & 0.18 \\
\hline $\mathrm{Ta}$ & 0.04 & 0.08 & 0.01 & 0.03 & 0.02 & 0.01 & 0.04 & 0.04 & 0.02 & 0.03 & 0.02 & 0.02 \\
\hline $\mathrm{Pb}$ & 0.99 & 2.76 & 0.03 & 0.78 & 0.25 & 0.99 & 0.11 & 0.10 & 0.51 & 0.05 & 0.36 & 0.04 \\
\hline Th & 0.01 & 0.03 & 0.01 & 0.03 & 0.01 & 0.01 & 0.04 & 0.03 & 0.01 & 0.02 & 0.02 & 0.01 \\
\hline $\mathrm{U}$ & 0.01 & 0.19 & 0.02 & 0.04 & 0.03 & 0.03 & 0.07 & 0.04 & 0.05 & 0.04 & 0.05 & 0.03 \\
\hline
\end{tabular}


Table 9 - Compatible elements zone in augite crystals. Blank = not analyzed.

\begin{tabular}{|c|c|c|c|c|c|c|c|c|c|c|c|c|}
\hline \multirow{2}{*}{$\frac{\text { Element }}{\text { Zone }}$} & \multicolumn{3}{|c|}{$\mathrm{Sc}$} & \multicolumn{3}{|c|}{$\mathrm{Co}$} & \multicolumn{3}{|c|}{$\mathrm{Ni}$} & \multicolumn{3}{|c|}{$\mathrm{Zn}$} \\
\hline & Core & Interm & Rim & Core & Interm & Rim & Core & Interm & Rim & Core & Interm & Rim \\
\hline WW223A & 2.52 & & & 2.32 & & & 19.18 & & & 2.43 & & \\
\hline P6220B & 2.51 & 2.61 & 3.34 & 1.3 & 1.38 & 1.7 & 11.56 & 10.44 & 7 & 0.9 & 1.16 & 1.73 \\
\hline TM134 & 2.83 & 3.13 & 2.89 & 1.29 & 1.32 & 1.39 & 3.11 & 2.71 & 1.7 & & $<0.9$ & \\
\hline TM139 & 5.33 & 5 & 4.59 & 1.86 & 1.71 & 1.86 & 49.4 & 37.45 & 20.33 & & $<0.7$ & \\
\hline TM148 & 5.52 & 5.52 & 5.96 & 1.88 & 2.41 & 2.2 & 145.5 & 100.05 & 49.8 & & $<0.9$ & \\
\hline KN161 & 3.3 & 3.06 & & 1.28 & 1.45 & & 6.66 & 4.68 & \multicolumn{4}{|c|}{$<0.7$} \\
\hline KN164 & 2.87 & 2.92 & & 1.45 & 1.69 & & 3.07 & 2.64 & \multicolumn{4}{|c|}{$<0.6$} \\
\hline KN169* & 3.39 & 3.51 & & 1.68 & 1.79 & & 3.34 & 2.91 & & 0.8 & 1.2 & \\
\hline KN171 & 3.47 & 3.73 & & 1.77 & 1.58 & & 5.68 & 4.52 & 5.1 & \multicolumn{2}{|c|}{$<0.7$} & \\
\hline TM102 & 2.95 & 3.31 & 3.24 & 0.92 & 1.03 & 0.94 & 2.51 & 2.28 & 1.78 & 0.45 & 0.45 & 1.34 \\
\hline TM105 & 3.41 & 3.58 & & 1.4 & 1.27 & & 25 & 16.67 & & \multicolumn{2}{|c|}{$<0.6$} & \\
\hline Element & \multicolumn{3}{|c|}{$\mathrm{V}$} & \multicolumn{3}{|c|}{$\mathrm{Cr}$} & \multicolumn{3}{|c|}{$\mathrm{Pb}$} & \multicolumn{3}{|c|}{$\mathrm{Tm}$} \\
\hline Zone & Core & Interm & $\operatorname{Rim}$ & Core & Interm & Rim & Core & Interm & Rim & Core & Interm & Rim \\
\hline WW223A & \multicolumn{3}{|l|}{$<0.8$} & & & & \multicolumn{2}{|l|}{0.99} & & \multicolumn{2}{|l|}{$<0.3$} & \\
\hline P6220B & 1.12 & 1.25 & 1.39 & & & & 3.3 & 2.77 & 2.2 & 0.74 & 0.96 & 1.04 \\
\hline TM134 & 0.94 & 1.04 & 0.86 & 0.55 & 1.64 & 0.11 & \multicolumn{3}{|c|}{$<0.5$} & \multicolumn{3}{|c|}{$<0.6$} \\
\hline TM139 & 2.68 & 2.38 & 1.82 & & $<0.1$ & & \multicolumn{3}{|c|}{$<0.03$} & \multicolumn{3}{|c|}{$<0.6$} \\
\hline TM148 & 2.37 & 2.32 & 1.07 & 23.39 & 2.12 & 0.17 & 1.05 & 1.19 & 0.73 & \multicolumn{3}{|c|}{$<0.9$} \\
\hline KN161 & 1.56 & 1.33 & & 9.26 & 1.45 & & \multicolumn{2}{|c|}{$<0.1$} & & \multicolumn{2}{|c|}{$<0.7$} & \\
\hline KN164 & 1.49 & 1.46 & & 4.92 & 1.07 & & & & & & & \\
\hline KN169* & & & & & & & & & & 0.65 & 1.1 & \\
\hline KN171 & 1.28 & 1.17 & & & & & & & & & & \\
\hline TM102 & & $<0.9$ & & 8.83 & 4.32 & 0.39 & & $<0.5$ & & & $<0.8$ & \\
\hline TM105 & & & & & & & & & & & & \\
\hline Element & & $\mathrm{Lu}$ & & & $\mathrm{Yb}$ & & & Но & & & Er & \\
\hline Zone & Core & Interm & $\operatorname{Rim}$ & Core & Interm & Rim & Core & Interm & Rim & Core & Interm & Rim \\
\hline WW223A & $<0.4$ & & & $<0.5$ & & & $<0.3$ & & & $<0.4$ & & \\
\hline P6220B & 0.93 & 1.2 & 0.99 & 0.98 & 1.18 & 0.98 & & $<1$ & & 0.76 & 0.87 & 1.05 \\
\hline TM134 & & $<0.7$ & & & $<0.7$ & & & $<0.7$ & & & $<0.7$ & \\
\hline TM139 & & $<0.8$ & & & $<0.7$ & & & $<0.7$ & & & $<0.7$ & \\
\hline TM148 & 0.49 & 0.76 & 1.27 & & $<0.9$ & & 0.39 & 0.54 & 1.1 & 0.43 & 0.57 & 1.07 \\
\hline KN161 & & & & & & & & & & & & \\
\hline KN164 & & & & & & & & & & & & \\
\hline KN169* & 0.78 & 1.34 & & 0.72 & 1.14 & & 0.71 & 1.1 & & 0.69 & 1 & \\
\hline KN171 & & & & & & & & & & & & \\
\hline TM102 & & $<0.8$ & & & $<0.7$ & & & $<0.8$ & & & $<0.6$ & \\
\hline TM105 & 0.69 & 1.11 & & & & & & & & & & \\
\hline
\end{tabular}

${ }^{*} \mathrm{KN} 169(\mathrm{Y}$ core $=0.65, \mathrm{Y}$ interm $=1.10, \mathrm{Y}$ rim $=0,87$; Dy core $=0.62$, Dy interm $=1.07$, Dy rim $=0.84)$. 
Table 10 - Compatible elements zone in pigeonite crystals. Blank $=$ not analyzed

\begin{tabular}{|c|c|c|c|c|c|c|c|c|c|c|c|c|}
\hline \multirow{2}{*}{$\frac{\text { Element }}{\text { Zone }}$} & \multicolumn{3}{|c|}{$\mathrm{Sc}$} & \multicolumn{3}{|c|}{$\mathrm{Co}$} & \multicolumn{3}{|c|}{$\mathrm{Ni}$} & \multicolumn{3}{|c|}{$\mathrm{Zn}$} \\
\hline & Core & Interm & Rim & Core & Interm & Rim & Core & Interm & Rim & Core & Interm & Rim \\
\hline WW223A & 2.16 & 2.36 & & 2.74 & 2.66 & & 13.48 & 12.08 & & 2.92 & 3.12 & \\
\hline KN164 & 1.42 & 1.5 & & 2.56 & 2.57 & & 4.11 & 3.58 & & 1.26 & 1.38 & \\
\hline KN171 & 1.23 & & & 2.02 & & & 13.25 & & & $<0.9$ & & \\
\hline TM102 & & & 1.45 & & & 1.87 & & & 2.11 & & & 1.51 \\
\hline TM105 & & & 1.56 & & & 1.9 & & & 17 & & & 1.78 \\
\hline Element & & $\mathrm{Pb}$ & & & $\mathrm{Cr}$ & & & $\mathrm{Lu}$ & & & & \\
\hline Zone & Core & Interm & Rim & Core & Interm & $\operatorname{Rim}$ & Core & Interm & $\operatorname{Rim}$ & & & \\
\hline WW223A & 1.29 & 1.06 & & & & & & & & & & \\
\hline KN164 & & & & & & & & & & & & \\
\hline KN171 & $<0.2$ & & & 3.47 & & & $<0.1$ & & & & & \\
\hline TM102 & & & $<0.1$ & & & $<0.1$ & & & $<0.6$ & & & \\
\hline TM105 & & & & & & & & & 1.13 & & & \\
\hline
\end{tabular}

Acknowledgements The first author wishes to thank CNPq and CAPES for the doctoral scholarship in Brazil and Germany, PRONEX-FAPERGS/ CNPq ("Strategic Minerals") for financial support.
CPRM (Geological Survey Brazil) provided support and most of the sill samples. Stuttgart University provided Electron Microprobe facility and overall support.

\section{References}

Arth J.G. 1976. Behaviour of trace elements during magmatic processes - a summary of theorical models and their applications. J. Res. U.S. Geol. Surv, 4:41-47.

BeattieP. 1993. The effect of partial melting of spinel peridotite on uranium series disequilibria: constraints from partitioning studies. Earth and Planetary Science Letters, 177:379-391. DOI: 10.1016/0012-821X(93)90091-M.

Bellieni G., Comin-Chiaramonti P., Marques L.S., Melfi A.J., Nardy A.J.R., Paratrechas C., Le Bas, M.J., Le Maitre R.W., Streckeisen A. Zannetin B. 1986. A chemical classification of volcanic rocks based on total alkali-silica diagram. Journal of Petrology, 27:745-750.

Benjamin T., Heuser W.R., Burnett D.S. 1978. Laboratory studies of Actinide Partitioning Relevant to 244 PU Chronometry. In: Lunar and Planetary Institute, Lunar and Planetary Science Conference, 9, Proceedings Paper, p. 1.393-1.406.

Best M.G. 2003. Igneous and Metamorphic Petrology. 2.ed., Massachusetts, Blackwell Science Ltd., 729 p.

Bindeman I.N., Davis A.M., Drake M.J. 1998. Ion microprobe study of plagioclases-basalt partition experiments at natural concentration levels of trace elements. Geochimica et Cosmochimica Acta., 62(7):1.175-1.193. DOI: 10.1016/S0016-7037(98)00047-7.

Bougault H. \& Hekinian R. 1974. Rift valley in the Atlantic Ocean near 36 degrees $50^{\prime} \mathrm{N}$; petrology and geochemistry of basalt rocks. Earth and Planetary Science Letters, 24(2):249-261. DOI: 10.1016/0012$821 \mathrm{X}(74) 90103-4$.

Bowen N.L. 1928. The evolution of the igneous rocks. Princeton, Princeton University Press, 334 p.
Dale I.M. \& Henderson P. 1972. The Partition of Transition Elements in Phenocryst-bearing Basalts and the Implications about Melt Structure. In: Geological Survey of Canada, International Geological Congress, 24, Sector 10, p. 105-111.

Deer A.A.W., Howie R.A., Zussman J. 2003. Minerais constituintes das rochas - uma introdução. Lisboa, Longmans, Green and Co. Ltda., 652 p.

Dostal J., Dupuy C., Carron J.P., Dekerneizon M.., Maury R.C. 1983. Partition-Coefficients of Trace-Elements - Application to Volcanic-Rocks of St-Vincent, WestIndies. Geochimica et Cosmochimica Acta, 47(3):525533. DOI: 10.1016/0016-7037(83)90275-2.

Drake M.J. \& Weill D.F. 1975. Partition of Sr, Ba, Ca, Y, Eu2+, $\mathrm{Eu} 3+$, and Other Ree between Plagioclases Feldspar and Magmatic Liquid - Experimental Study. Geochimica et Cosmochimica Acta, 39(5):689-712. DOI: 10.1016/00167037(75)90011-3.

Duke J.M. 1976. Distribution of the period four transition elements among olivine, calcic clinopyroxene and mafic silicate liquid; experimental results. Journal of Petrology, 17(4):499-521.

Dunn T. \& Sen C. 1994. Mineral/Matrix Partition-Coefficients for Ortho-Pyroxene, Plagioclases, and Olivine in Basaltic to Andesitic Systems - a Combined Analytical and Experimental-Study. Geochimica et Cosmochimica Acta, 58(2):717-733. DOI: 10.1016/0016-7037(94)90501-0.

Ewart A., Bryan W.B., Gill J.B. 1973. Mineralogy and Geochemistry of the Younger Volcanic Islands of Tonga, S. W. Pacific. Journal of Petrology, 14(3):429-465.

Forsythe L.M., Nielsen R.L., Fisk M.R. 1994. High-FieldStrength Element Partitioning between Pyroxene and 
Basaltic to Dacitic Magmas. Chemical Geology, 117(14):107-125. DOI: 10.1016/0009-2541(94)90124-4.

Frey F.A. 1969. Rare earth abundances in a high-temperature peridotite intrusion. Geochimica et Cosmochimica Acta, 33(11):1.429-1.447. DOI: 10.1016/00167037(69)90183-5.

Fujimaki H., Tatsumoto M., Aoki K. 1984. Partition coefficients of $\mathrm{Hf}, \mathrm{Zr}$, and REE between phenocrysts and groundmasses. In: Lunar and Planetary Institute, Lunar and Planetary Science Conference, 14, Proceedings Paper, p. B662-B672.

Gaetani G.A. \& Grove T.L. 1995. Partitioning of Rare-Earth Elements between Clinopyroxene and Silicate Melt Crystal-Chemical Controls. Geochimica et Cosmochimica Acta, 59(10):1.951-1.962. DOI: 10.1016/001670379(50)01190-.

Hack P.J, Nielsen R.L., Johnston A.D. 1994. Experimentally determined rare-Earth element and $\mathrm{Y}$ partitioning behavior between clinopyroxene and basaltic liquids at pressures up to $20 \mathrm{kbar}$. Chemical Geology, 117:89-105. DOI: 10.1016/0009-2541(94)90123-6.

Hart S.R. \& Brooks C. 1974. Clinopyroxene-matrix partitioning of $\mathrm{K}, \mathrm{Rb}, \mathrm{Cs}, \mathrm{Sr}$ and $\mathrm{Ba}$. Geochimica et Cosmochimica Acta, 38:1.799-1.806. DOI: 10.1016/00167037(74)90163-X.

Hart S.R. \& Dunn T. 1993. Experimental cpx/melt partitioning of 24 trace elements. Contributions to Mineralogy and Petrology, 113:1-8.

Hauri E.H., Wagner T.P., Grove T.L. 1994. Experimental and natural partitioning of $\mathrm{Th}, \mathrm{U}, \mathrm{Pb}$ and other trace elements between garnet, clinopyroxene and basaltic melts. Chemical Geology, 117:149-166. DOI: 10.1016/00092541(94)90126-0.

Irving A.J. \& Frey F.A. 1984. Trace-Element Abundances in Megacrysts and Their Host Basalts - Constraints on Partition-Coefficients and Megacryst Genesis. Geochimica et Cosmochimica Acta, 48(6):1.201-1.221. DOI: 10.1016/0016-7037(84)90056-5.

Irvine T.N. \& Baragar W.R.A. 1971. A guide to the chemical classification of the common volcanic rocks. Canadian Journal of Earth Sciences, 8:523-548.

Jenner G.A., Foley S.F., Jackson S.E., Green T.H., Fryer B.J., Longerich H.P. 1993. Determination of partition coefficients for trace elements in high pressuretemperature experimental run products by laser ablation microprobe-inductively coupled plasmamass spectrometry (LAM-ICP-MS). Geochimica et Cosmochimica Acta, 57(23-24):5.099-5.103.

Kravuchuk I.K., Chernysheva I., Urosov S. 1981. Element distribution between plagioclases and groundmass as an indicator for crystallization conditions of the basalts in the southern vent of Tolbachik. Geochemistry International, 17:18-24.

Larsen L.M. 1979. Distribution of Ree and Other TraceElements between Phenocrysts and Peralkaline Undersaturated Magmas, Exemplified by Rocks from the Gardar Igneous Province, South Greenland. Lithos, 12(4):303-315. DOI: 10.1016/0024-4937(79)90022-7.

Lindsley D. 1983. Pyroxene thermometry. American Mineralogist, 68:477-493.
Machado F.B. 2003. Geologia e possíveis zonas de efusão do magmatismo ácido cretácico da Bacia do Paraná. Monografia (TCC), Instituto de Geociências e Ciências Exatas, Universidade Estadual Paulista, Rio Claro, 124 p.

Matsui Y., Onuma N., Nagasawa H., Higuchi H., Banno S. 1977. Crystal structure control in trace element partition between crystal and magma. Tectonics, 100:315-324.

McCallum I.S. \& Charette M.P. 1978. Zr and $\mathrm{Nb}$ partition coefficients: implications for the genesis of mare basalts, kreep, and sea floor basalts. Geochimica et Cosmochimica Acta, 42:859-869. DOI: 10.1016/00167037(78)90098-4.

McKay G., Le L., Wagstaff J., Crozaz G. 1994. Experimental partitioning of rare Earth elements and strontium: constraints on petrogenesis and redox conditions during crystallization of Antarctic angrite Lewis Cliff 86010. Geochimica et Cosmochimica Acta, 58:2.911-2.919. DOI: 10.1016/0016-7037(94)90124-4.

McKenzie D. \& O’Nions R.K. 1991. Partial melt distributions from inversion of rare Earth element concentrations. Journal of Petrology, 32:1.021-1.091.

Melfi A.J., Piccirillo E.M., Nardy A.J.R. 1988. Geological and magmatic aspects of the Paraná Basin - an introduction. In: Piccirillo E.M. \& Melfi A.J. (eds.) The Mesozoic flood volcanism of the Paraná Basin: petrogenetic and geophysical aspects. São Paulo, Instituto Astronômico e Geofísico, p. 1-14.

Morimoto N. 1988. Nomenclature of Pyroxene. Min. Mag., 52:535-50.

Nagasawa H. 1973. Rare-Earth distribution in alkali rocks from Oki-Dogo Island, Japan. Contributions to Mineralogy and Petrology, 39:301-308.

Nardy A.J.R., Oliveira M.A.F., De Betancourt R.H.S., Verdugo D.R.H., Machado F.B. 2002. Geologia e estratigrafia da Formação Serra Geral. Rev. Geociências, 21:15-32.

Paster T.P., Schauwecker D.S., Haskin L.A. 1974. The behavior of some trace elements during solidification of the Skaergaard layered series. Geochimica et Cosmochimica Acta, 38(10):1.549-1.577. DOI: 10.1016/0016-7037(74)90174-4.

Pearce N.J.G., Perkins W.T., Westgate J.A., Gorton M.P., Jackson S.E., Neal C.R., Chenery S.P. 1997. A compilation of new and published major and trace element data for NIST SRM 610 and NIST SRM 612 glass reference materials. Geostandards Newsletter, 21(1):115-144.

Reid F. 1983. Origin of the Rhyolitic Rocks of the Taupo Volcanic Zone, New-Zealand. Journal of Volcanology and Geothermal Research, 15(4):315-338.

Ringwood A.E. 1970. Petrogenesis of Apollo 11 basalts and implications for lunar origin. Journal of Geophysical Research, 75(32):6.453-6.479.

Schnetzler C.C. \& Philpotts J.A. 1970. Partition coefficients of rare-earth elements between igneous matrix material and rock-forming mineral phenocrysts; II. Geochimica et Cosmochimica Acta, 34(3):331-340. DOI: 10.1016/0016-7037(70)90110-9.

Skulski T., Minarik W., Watson E.B. 1994. HighPressure Experimental Trace-Element Partitioning 
between Clinopyroxene and Basaltic Melts. Chemical Geology, 117(1-4):127-147. DOI: 10.1016/00092541(94)90125-2.

Sobolev A.V., Migdisov A.A., Portnyagin M.V. 1996. Incompatible element partitioning between clinopyroxene and basalt liquid revealed by the study of melt inclusions in minerals from Troodos lavas, Cyprus. Petrology, 4(3):307-317.

Sun C.O., Williams R.J., Sun S.S. 1974. Distribution coefficients of $\mathrm{Eu}$ and $\mathrm{Sr}$ for plagioclases-liquid and clinopyroxene-liquid equilibria in oceanic ridge basalt; an experimental study. Geochimica et Cosmochimica Acta, 38(9):1.415-1.433. DOI: 10.1016/00167037(74)90096-9.

Villemant B., Jaffrezic H., Joron J.L., Treuil M. 1981. Distribution Coefficients of Major and Trace-Elements
- Fractional Crystallization in the Alkali Basalt Series of Chaine-Des-Puys (Massif Central, France). Geochimica et Cosmochimica Acta, 45(11):1.997-2.016. DOI: 10.1016/0016-7037(81)90055-7.

Watson E.B., Othman D.B., Luck J.M., Hofmann A.W. 1987. Partitioning of $\mathrm{U}, \mathrm{Pb}, \mathrm{Cs}, \mathrm{Yb}, \mathrm{Hf}, \mathrm{Re}$ and Os between Chromian Diopsidic Pyroxene and Haplobasaltic Liquid. Chemical Geology, 62(3-4):191-208. DOI: 10.1016/0009-2541(87)90085-4.

Zanettin B. 1984. Proposed new chemical classification of volcanic rocks. Episodes, 7:19-20.

Manuscrito ID 16503

Submetido em 31 de dezembro de 2009 Aceito em 04 de outubro de 2011 